\title{
Analysis of fluid systems: stability, receptivity, sensitivity
}

\author{
Peter J. Schmid \\ Department of Mathematics \\ Imperial College London \\ London, SW7 2AZ, United Kingdom \\ peter.schmid@imperial.ac.uk \\ Luca Brandt \\ Linné FLOW Centre \\ Department of Mechanics, Royal Institute of Technology (KTH) \\ SE-10044 Stockholm, Sweden \\ Email: luca@mech.kth.se
}

This article presents techniques for the analysis of fluid systems. It adopts an optimization-based point of view, formulating common concepts such as stability and receptivity in terms of a cost functional to be optimized subject to constraints given by the governing equations. This approach differs significantly from eigenvalue-based methods that cover the time-asymptotic limit for stability problems or the resonant limit for receptivity problems. Formal substitution of the solution operator for linear time-invariant systems results in the matrix exponential norm and the resolvent norm as measures to assess the optimal response to initial conditions or external harmonic forcing. The optimization-based approach can be extended by introducing adjoint variables that enforce governing equations and constraints. This step allows the analysis of far more general fluid systems, such as time-varying and nonlinear flows, and the investigation of wavemaker regions, structural sensitivities and passive control strategies.

\section{Introduction and motivation}

Fluid systems are often described and characterized by their stability or receptivity behavior. Perturbations of infinitesimal amplitude that grow when superimposed on an equilibrium state of the flow render the base flow unstable; similarly, a flow that responds strongly when harmonically forced by an external excitation is referred to as receptive to this particular driving. Standard mathematical techniques have been devised to describe these fundamental questions of fluid dynamics: eigenvalue analysis for stability problems, and the resonance concept for receptivity problems. If the linearized equations exhibit at least one eigenvalue in the unstable half-plane, an instability is deduced; if the forcing frequency coincides with one of the eigenvalues of the lin- earized equations, a resonance is present in the flow.

Even though these techniques are valuable quantitative tools for the description of fluid problems, they have been found inadequate to account for the full behavior of many fluid systems. A property of the underlying equations, known as nonnormality, allows for a far richer linear behavior than what can be measured by eigenvalues or resonances alone. By recasting the questions of instability and receptivity into a framework based on constrained optimization, new tools and viewpoints arise that present a more complete picture of linear perturbation dynamics for fluid flows. An even more effective framework emerges by the transformation of the constrained into an unconstrained optimization problems using adjoint variables (or Lagrange multipliers). The introduction of adjoint variables may, at first glance, appear as a mathematical device to enforce constraints; their interpretation as sensitivity measures or cost-functional gradients, however, gives them a physical meaning that can readily be used to assess important aspects of the flow behavior, to quantify robustness or to design passive control strategies.

The objective of this article is to present a suite of techniques for the analysis of fluid behavior for simple shear flows, but also to advocate an approach for this analysis that exceeds standard methods and harnesses the capability and potential of modern mathematical techniques arising from an optimization and system-theoretic framework. This article is based on a tutorial given at the Nordita workshop on "Stability and Transition" which took place in Stockholm from May 6-31, 2013. The Matlab codes used in this tutorial are available from the journal website and cover the majority of the concepts (and figures) treated in this article. 
(a)

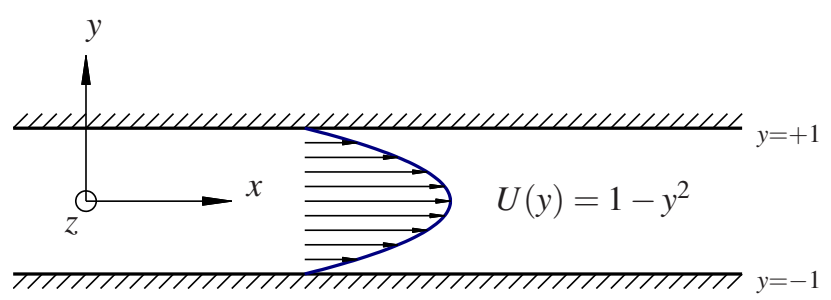

(b)

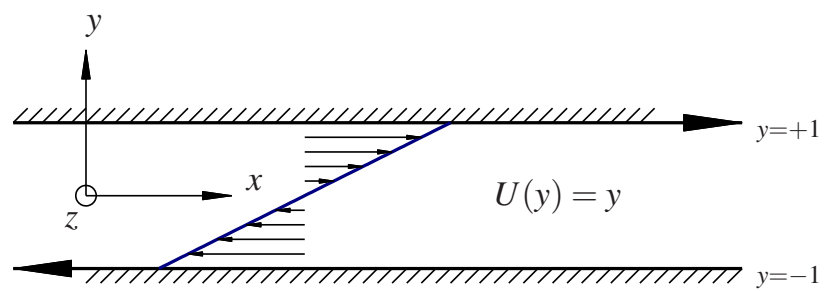

Fig. 1. Sketch of the two flow configurations, the coordinate system and the base flow profiles. (a) Plane Poiseuille flow, and (b) plane Couette flow.

\section{The governing equations}

Even though the tools and techniques in this article readily apply to more complex flows, for sake of clarity we will consider the flow of an incompressible fluid confined by two walls. Two cases will be be treated: (i) the pressure-driven flow between two resting plates yielding a parabolic baseflow velocity profile (i.e., plane Poiseuille flow), and (ii) the flow induced by the two plates moving in-plane in opposite directions by the same speed producing a linear base-flow velocity profile (i.e., plane Couette flow). In either case, the base flow is given by the streamwise velocity component $U(y)$ which only varies in the normal (plate-to-plate) direction $y$. A sketch of the two flow cases, together with the coordinate system, is given in figure 1 .

Linearizing the incompressible Navier-Stokes equations about the base flow $U(y)$ yields the following system of equations

$$
\begin{aligned}
\frac{\partial u}{\partial t}+U \frac{\partial u}{\partial x}+v U^{\prime} & =-\frac{\partial p}{\partial x}+\frac{1}{R e} \nabla^{2} u+f_{u} \\
\frac{\partial v}{\partial t}+U \frac{\partial v}{\partial x} & =-\frac{\partial p}{\partial y}+\frac{1}{R e} \nabla^{2} v+f_{v} \\
\frac{\partial w}{\partial t}+U \frac{\partial w}{\partial x} & =-\frac{\partial p}{\partial z}+\frac{1}{R e} \nabla^{2} w+f_{w} \\
\frac{\partial u}{\partial x}+\frac{\partial v}{\partial y}+\frac{\partial w}{\partial z} & =0
\end{aligned}
$$

for the perturbation velocities $(u, v, w)$ and the perturbation pressure $p$, where $\nabla^{2}$ stands for the standard Cartesian Laplace operator and ' denotes differentiation with respect to $y$. To each of the momentum equation, we have added an external driving term which will later be used in receptivity studies. The above equations have been nondimensional- ized by the channel half-height and the center-line velocity (in the Poiseuille case) or the speed of the moving wall (in the Couette case). These nondimensionalizations produce a Reynolds number as follows: $R e=U h / v$, with $v$ as the kinematic viscosity. No-slip boundary conditions on the wall, i.e., $u=v=w=0$ at $y= \pm 1$, and appropriate initial conditions complete the evolution problem for the perturbations. Both flow configurations are assumed infinite in the streamwise $(x)$ and spanwise $(z)$ directions. We also observe that the governing equations (1) have coefficients that are constant in these two coordinate directions. As a consequence, this allows the application of a Fourier transform in these directions, which is equivalent to assuming solutions of the form

$$
\left(\begin{array}{l}
u(x, y, z, t) \\
v(x, y, z, t) \\
w(x, y, z, t) \\
p(x, y, z, t)
\end{array}\right)=\left(\begin{array}{c}
\hat{u}(y, t) \\
\hat{v}(y, t) \\
\hat{w}(y, t) \\
\hat{p}(y, t)
\end{array}\right)_{(\alpha, \beta)} \exp (i \alpha x+i \beta z) .
$$

This mathematical step introduces the streamwise and spanwise wavenumbers $\alpha$ and $\beta$ and simplifies the governing equations to

$$
\begin{aligned}
\frac{\partial \hat{u}}{\partial t}+i \alpha U \hat{u}+U^{\prime} \hat{v} & =-i \alpha \hat{p}+\frac{1}{R e}\left(\mathcal{D}^{2}-k^{2}\right) \hat{u}+\hat{f}_{u} \\
\frac{\partial \hat{v}}{\partial t}+i \alpha U \hat{v} & =-\mathcal{D} \hat{p}+\frac{1}{R e}\left(\mathcal{D}^{2}-k^{2}\right) \hat{v}+\hat{f}_{v} \\
\frac{\partial \hat{w}}{\partial t}+i \alpha U \hat{w} & =-i \beta \hat{p}+\frac{1}{R e}\left(\mathcal{D}^{2}-k^{2}\right) \hat{w}+\hat{f}_{w} \\
i \alpha \hat{u}+\mathcal{D} \hat{v}+i \beta \hat{w} & =0
\end{aligned}
$$

where $\mathcal{D}=\partial / \partial y$ represents differentiation with respect to $y$, and the total wavenumber $k$ is defined through $k^{2}=\alpha^{2}+\beta^{2}$. In a final step we eliminate the pressure from the above equations. This is accomplished by a variable transformation which introduces the normal velocity $\hat{v}$ and the normal vorticity $\hat{\eta}=i \beta \hat{u}-i \alpha \hat{w}$ in lieu of the primitive variables $\hat{u}, \hat{v}, \hat{w}, \hat{p}$. Mathematically, we proceed in two steps. First, we multiply (3a) by i $\beta$ and subtract it from $i \alpha$ times (3c) which yields an equation for $\hat{\eta}$. This step is equivalent to taking the curl $\nabla \times$ of the governing equations and extracting the normal $y$-component; it naturally eliminates the pressure gradient term. The second step consist of multiplying (3a,3c) by $i \alpha$ and $i \beta$, respectively, and adding the two equations. Using (3d) and the definition of the normal vorticity $\hat{\eta}$, an expression for the pressure $\hat{p}$ can be derived that solely depends on the normal velocity $\hat{v}$. This latter expression is then resubstituted into ( $3 \mathrm{~b}$ ) to eliminate the remaining pressure term $\mathcal{D} \hat{p}$. After these algebraic manipulations we arrive at a system of two evolution equations (for the normal velocity $\hat{v}$ and the normal vorticity equation $\hat{\eta}$ ) which reads 


$$
\begin{aligned}
\mathcal{M} \frac{\partial \hat{v}}{\partial t}+i \alpha U \mathcal{M} \hat{v}+i \alpha U^{\prime \prime} \hat{v}+\frac{1}{R e} \mathcal{M}^{2} \hat{v} & =\hat{g}_{v} \\
\frac{\partial \hat{\eta}}{\partial t}+i \alpha U \hat{\eta}+\frac{1}{R e} \mathcal{M} \hat{\eta} & =-i \beta U^{\prime} \hat{v}+\hat{g}_{\eta}(
\end{aligned}
$$

with $\mathcal{M}=k^{2}-\mathcal{D}^{2}$. The above equations have to be supplemented by boundary conditions. Requiring no-slip conditions at the wall, it is straightforward to derive the following boundary conditions for $\hat{v}$ and $\hat{\eta}$.

$$
\mathcal{D} \hat{v}( \pm 1)=\hat{v}( \pm 1)=\hat{\eta}( \pm 1)=0
$$

To conclude the derivation of the governing equations, we discretize the above partial differential equation in the wallnormal $y$-direction. Even though a variety of numerical techniques are available, we choose a spectral technique using Chebyshev polynomials and replace the continuous wall-normal differentiation operator $\mathcal{D}$ by the Chebyshevdifferentiation matrix D. Consequently, the dependent variables $\hat{v}$ and $\hat{\eta}$ transform into column vectors $\mathbf{v}, \eta$ containing the values of the wall-normal velocity and vorticity at the Chebyshev collocation points. After these steps we end up with a system of ordinary differential equations in time which reads

$$
\frac{d}{d t}\left(\begin{array}{l}
\mathbf{v} \\
\eta
\end{array}\right)=\underbrace{\left(\begin{array}{cc}
\mathrm{L}_{\mathrm{OS}} & 0 \\
\mathrm{~L}_{\mathrm{C}} & \mathrm{L}_{\mathrm{SQ}}
\end{array}\right)}_{\mathrm{L}}\left(\begin{array}{l}
\mathbf{v} \\
\eta
\end{array}\right)+\mathrm{B}\left(\begin{array}{l}
\mathbf{f}_{u} \\
\mathbf{f}_{v} \\
\mathbf{f}_{w}
\end{array}\right)
$$

with

$$
\begin{aligned}
\mathrm{L}_{\mathrm{OS}} & =\mathrm{M}^{-1}\left(-i \alpha U \mathrm{M}-i \alpha U^{\prime \prime}-\frac{1}{R e} \mathrm{M}^{2}\right), \\
\mathrm{L}_{\mathrm{SQ}} & =-i \alpha U-\frac{1}{R e} \mathrm{M}, \\
\mathrm{L}_{\mathrm{C}} & =-i \beta U^{\prime}, \\
\mathrm{B} & =\left(\begin{array}{ccc}
i \alpha \mathrm{M}^{-1} \mathrm{D} & \mathrm{M}^{-1} k^{2} & i \beta \mathrm{M}^{-1} \mathrm{D} \\
i \beta & 0 & -i \alpha
\end{array}\right) .
\end{aligned}
$$

We notice that the normal-velocity equation is homogeneous, while the normal-vorticity equation is driven by the normal velocity in the presence of base-flow shear $U^{\prime}$ and as long as $\beta \neq 0$. This observation will play a very important role in the analysis to follow. Upon further introducing the composite vector of unknowns $\mathbf{q}=(\mathbf{v}, \eta)^{T}$, as well as the composite forcing $\mathbf{f}=\left(\mathbf{f}_{u}, \mathbf{f}_{v}, \mathbf{f}_{w}\right)^{T}$, the above set of equations then simplifies to

$$
\frac{d}{d t} \mathbf{q}=\mathrm{L} \mathbf{q}+\mathrm{B} \mathbf{f}
$$

which will form the foundation for the analysis to follow.

Even though the bulk of this article is concerned with the stability, receptivity and sensitivity analysis of wallbounded incompressible shear flow (in particular, with plane Poiseuille and plane Couette flow), it will be instructive at times to consider a small model problem with two degrees of freedom that mimics many of the features observed in the full flow equations. In accordance with the governing equations derived above we propose the simple equation

$$
\frac{d}{d t}\left(\begin{array}{l}
q_{1} \\
q_{2}
\end{array}\right)=\underbrace{\left(\begin{array}{cc}
\frac{1}{100}-\frac{1}{R e} & 0 \\
\mu & -\frac{2}{R e}
\end{array}\right)}_{\mathrm{A}}\left(\begin{array}{l}
q_{1} \\
q_{2}
\end{array}\right)
$$

for the temporal evolution of two variables $q_{1}$ and $q_{2}$. As is immediately obvious, it closely resembles the structure of (6): in particular, the driving of the second variable by the first has been incorporated via the parameter $\mu$. An explicit dependence on a parameter (taken as the Reynolds number $R e$ ) has also been introduced. A quick analysis of this system as to its eigenvalues gives a first (though incomplete) glance of the perturbation dynamics. The particular form of the system matrix $A$ allows us to determine the eigenvalues as $\lambda_{1}=1 / 100-1 / \operatorname{Re}$ and $\lambda_{2}=-2 / R e$; the first one changes sign at a critical value of the Reynolds number $\left(R e_{c r i t}=100\right)$, the second one is always negative. For $\operatorname{Re}<100$, we thus have a configuration with stable eigenvalues $\lambda_{1,2}$. Analogous findings can be derived for the full system matrix $L$ for plane Poiseuille flow (with a critical Reynolds number of $R e_{\text {crit }}=5772.2$ ).

\section{Stability analysis of fluid systems}

Before continuing with the stability analysis of fluid systems, it is necessary to give a definition of stability. Traditionally this has been done following the concept of Lyapunov stability. An equilibrium state has to be defined first, after which the system is perturbed around this state. If the system returns back to the equilibrium state, it is deemed stable; if the system diverges from the equilibrium state, the system (or, more precisely, this particular equilibrium state) is regarded as unstable. In the definition of Lyapunov stability, an infinite time horizon is allowed for the return to equilibrium.

In many fluid systems where stability issues play an important role an infinite time horizon does not account for the many time-scales that characterize local fluid processes. In fact, it can be argued that most dynamic processes in wallbounded shear flows occur on a finite time-scale, often related to, e.g., a characteristic eddy turn-over time or the lifetime of coherent structures involved in the process. A stability definition that is based on an infinite time horizon seems to run counter to the observation of finite-time processes. For 
this reason, we will abandon the concept of Lyapunov stability and define stability as the amplification of initial perturbation energy over a prescribed time-interval [1,2]; this definition reintroduces the time variable as a parameter. The amplification of initial energy of course depends on the initial condition. This dependence can be eliminated by optimizing over all permissible initial conditions and accepting the maximum as the optimal energy amplification. Mathematically, we can write

$$
G(t)=\max _{\mathbf{q}_{0}} \frac{E(\mathbf{q}(t))}{E\left(\mathbf{q}_{0}\right)}
$$

where $E(\mathbf{q})$ denotes the kinetic energy of the perturbation $\mathbf{q}$. We will introduce a more general measure by defining the general norm of $\mathbf{q}$ as the quantity to be optimized. Other quantities that define a norm, such as, e.g., enstrophy or dissipation rate, are conceivable and may be more appropriate for specific applications or problem settings.

In anticipation of the analysis that follows below, we reformulate the above norm of the perturbations (in our case yielding the kinetic energy) and relate it to the common $L_{2}$ norm of a vector $\mathbf{q}$. The following simple transforms establish a connection between the energy norm $\|.\|_{E}$ and the standard (Euclidean) $L_{2}$-norm $\|.\|_{2}$.

$$
\begin{aligned}
E(\mathbf{q}) & =\|\mathbf{q}\|_{E}^{2}=\langle\mathbf{q}, \mathbf{q}\rangle_{E}=\mathbf{q}^{H} \mathbf{Q} \mathbf{q} \\
& =\mathbf{q}^{H} F^{H} \mathbf{F q}=\langle F \mathbf{q}, F \mathbf{q}\rangle_{2}=\|F \mathbf{q}\|_{2}^{2}
\end{aligned}
$$

The above expression introduces the energy weight matrix $Q$ which contains the proper weighting of the variables, in our case $\mathbf{v}$ and $\eta$ at the spectral collocation points, as well as the integration weights to evaluate the integral in the wallnormal direction from the lower to the upper plate. It follows from the fact that we express a positive quantity (energy norm), that the weight matrix $Q$ has to be positive definite. In this case, it can be decomposed into $\mathrm{Q}=\mathrm{F}^{H} \mathrm{~F}$ using a Cholesky decomposition. The energy norm of a perturbation $\mathbf{q}$ is thus equivalent to the $L_{2}$-norm of the vector Fq.

We further have to introduce the equivalent energy norm for matrices and, as before, relate it to the common $L_{2}$-norm. Using the definition of a vector-induced norm we easily find

$$
\|\mathrm{L}\|_{E}=\max _{\mathbf{q}} \frac{\|\mathrm{L} \mathbf{q}\|_{E}}{\|\mathbf{q}\|_{E}}=\max _{\mathbf{q}} \frac{\left\|\mathrm{FLF}^{-1} \mathrm{Fq}\right\|_{2}}{\|\mathrm{Fq}\|_{2}}=\left\|\mathrm{FLF}^{-1}\right\|_{2}
$$

It follows from the above expression that a simple similarity transformation using the Cholesky factor $F$ relates the energy norm to the $L_{2}$-norm for matrices.

\subsection{The matrix exponential norm}

We proceed by evaluating the ratio of perturbation energy at a given time $t$ to the initial energy maximized over all possible initial conditions. This ratio of output (the perturbation energy at $t$ ) to input (the perturbation energy at time $t=0$ ) will be taken as our measure of (in)stability over a given time interval $[0 t]$. Of course, the output $\mathbf{q}(t)$ is related to the initial condition $\mathbf{q}(0)=\mathbf{q}_{0}$ by equation (6) governing the evolution of initial conditions in time. We will neglect the forcing term $\mathbf{f}$ for this analysis. The governing equations are linear, time-invariant which allows us to state the formal solution in form of the matrix exponential according to

$$
\mathbf{q}(t)=\exp (t \mathrm{~L}) \mathbf{q}_{0}
$$

This expression links initial conditions to solutions of our governing equation at a later time. Substituting the above expression into our definition of energy amplification (10), we obtain

$$
G(t)=\max _{\mathbf{q}_{0}} \frac{\|\mathbf{q}(t)\|_{E}^{2}}{\left\|\mathbf{q}_{0}\right\|_{e}^{2}}=\max _{\mathbf{q}_{0}} \frac{\left\|\exp (t \mathrm{~L}) \mathbf{q}_{0}\right\|_{E}^{2}}{\left\|\mathbf{q}_{0}\right\|_{E}^{2}}=\|\exp (t \mathrm{~L})\|_{E}^{2}
$$

where the last step in the above expression has invoked the definition of a vector-induced matrix norm, taking care of the optimization over all initial conditions. The energy norm of the matrix exponential is thus the largest amplification of energy any initial perturbation can experience over a given time interval $[0 t]$. This expression can be evaluated for a variety of time horizons $t$, including values that are deemed characteristic of the time-scales imposed by the flow. The values of the corresponding matrix exponential norms give a first insight into the capability of the fluid system to optimally amplify perturbation energy contained in an initial condition over a limited time span.

It is important to realize that the energy amplification $G(t)$ is optimal over all possible initial conditions, but that for each chosen time span $t$ a different initial condition may yield the optimal gain $G(t)$. The curve $G(t)$ versus $t$ may thus be thought of as an envelope over optimal initial conditions; an initial condition at a chosen time horizon $t_{1}$ may not be optimal for a different time horizon $t_{2} \neq t_{1}$.

We will illustrate the energy amplication or gain $G(t)$ for the $2 \times 2$-model equation for $\mu=1$. In this case, the matrix exponential can be computed analytically. We obtain

$$
\exp (t \mathrm{~A})=\left(\begin{array}{cc}
\exp \left(t \lambda_{1}\right) & 0 \\
\frac{\exp \left(t \lambda_{1}\right)-\exp \left(t \lambda_{2}\right)}{\lambda_{1}-\lambda_{2}} & \exp \left(t \lambda_{2}\right)
\end{array}\right)
$$




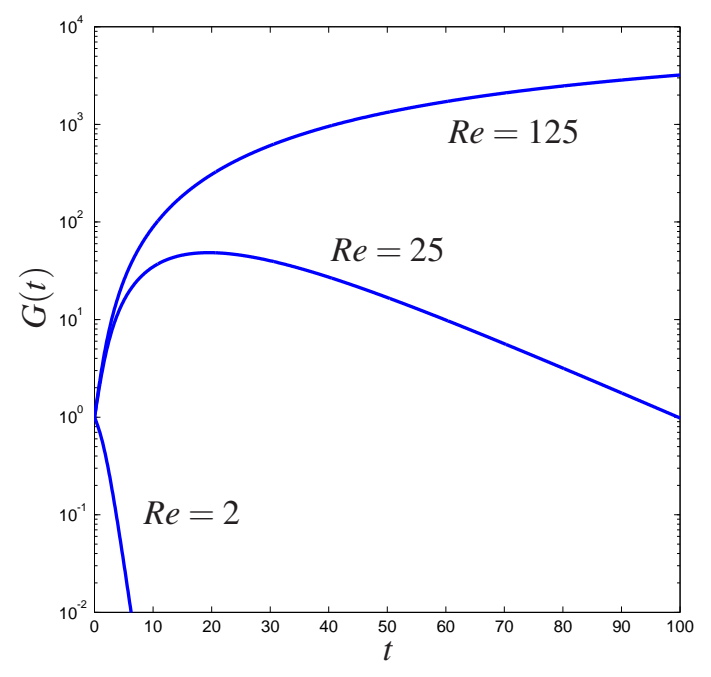

Fig. 2. Energy amplification $G(t)$ for the model problem for three different Reynolds numbers, showing monotonic decay (for $R e=2$ ), transient growth and asymptotic decay (for $R e=25$ ), and transient and asymptotic growth (for $\operatorname{Re}=125$ ).

$$
\begin{aligned}
G(t) & =\|\exp (t \mathrm{~A})\|_{2}^{2}=\frac{\operatorname{tr}}{2}+\sqrt{\frac{\operatorname{tr}^{2}}{4}-\operatorname{det}} \\
\operatorname{tr} & =\left(1+\frac{1}{\left(\lambda_{1}-\lambda_{2}\right)^{2}}\right)\left(\exp \left(2 \lambda_{1} t\right)+\exp \left(2 \lambda_{2} t\right)\right) \\
& -\frac{2 \sqrt{\operatorname{det}}}{\left(\lambda_{1}-\lambda_{2}\right)^{2}} \\
\operatorname{det} & =\exp \left(2\left(\lambda_{1}+\lambda_{2}\right) t\right)
\end{aligned}
$$

We choose a set of Reynolds number $R e=2,25,125$ and display $G(t)$ as a function of time in figure 2. We observe monotonic decay of $G(t)$ for the case of $R e=2$, a transient peak followed by exponential decay for $R e=25$, and for a supercritical Reynolds number $R e=125$ a strong amplification followed by exponential growth. In particular the case of $R e=25$ may come as a surprise, given the fact that at this Reynolds number the system matrix A had two eigenvalues with decaying real parts. We will next explore the origin of this transient growth in energy.

\subsection{The modal limit}

A closer look at the gain $G(t)$ involves an eigenvalue decomposition of $A$, given as $A=V \wedge V^{-1}$ with $V$ as the matrix containing the normalized eigenvectors as columns and $\Lambda$ as a diagonal matrix containing the corresponding eigenvalues. For the energy amplification or gain $G(t)$ we then have

$$
G(t)=\|\exp (t \mathrm{~A})\|^{2}=\left\|\mathrm{V} \exp (t \Lambda) \mathrm{V}^{-1}\right\|^{2} .
$$

The last expression makes clear that the eigenvalues contained in $\Lambda$ represent only one part of the gain $G(t)$, with the eigenvector matrix $\mathrm{V}$ and its inverse accounting for the remaining factors. Deducing the gain $G(t)$ from the eigenvalue matrix $\Lambda$ alone is only valid, if the similarity transformation given by $\mathrm{V}$ and its inverse does not alter the value of the norm. This is the case for unitary matrices $V$ as they represent pure rotations in vector space. In our case, orthogonal eigenvectors of $\mathrm{A}$ will result in a unitary $\mathrm{V}$. We conclude from this that $G(t)$ evolves according to the eigenvalue matrix $\Lambda$ for system matrices $A$ that have orthogonal eigenvectors. If this is not the case, eigenvalues alone do not fully describe the potential energy amplification that can take place in our system. System matrices A that result in non-orthogonal eigenvectors are known as nonnormal matrices, while matrices with orthogonal eigenvectors are referred to as normal.

The observation above suggests that short-time growth of perturbation energy is possible even though the system matrix has stable eigenvalues. The eigenvalue decomposition clearly shows that exponentially decaying solutions, given by the term $\exp (t \Lambda)$, can produce short-time growth in energy when superimposed non-orthogonally, given by $\mathrm{V}$ and $V^{-1}$. This fact is illustrated geometrically in figure 3 . In both subplots, we represent a unit-norm initial condition (the thick blue line) as a superposition of two eigenvectors; also in both cases, we assume that the eigenvalues along these eigendirections are real, distinct and negative, thus decaying to zero over time. On the left subplot, we chose orthogonal eigenvectors, and consequently the length of the initial condition shrinks monotonically to zero with a decay rate that is given by the larger of the two contractive eigenvalues. In the right subplot we perform the same exercise, with identical eigenvalues, this time, however, with non-orthogonal eigenvectors. We clearly observe that the initially unit-norm initial condition stretches before ultimately decaying to zero (again, with the decay rate of the least stable eigenvalue). Evaluating the norm (length) of the evolving initial condition, we observe transient growth in the nonnormal case and monotonic decay in the normal case.

A corollary to the above observation is that eigenvalues are an inherently time-asymptotic tool when dealing with nonnormal system matrices; they only accurately describe the complete perturbation dynamics (i.e., the perturbation dynamics for all times) for normal systems. We also conclude that Lyapunov stability (based on an infinite time horizon) is properly captured and evaluated by the eigenvalues of the underlying system matrix, but we have concluded earlier that finite-time processes in fluid systems call for a different approach, such as the one based on the matrix exponential.

Before proceeding to additional tools for the analysis of finite-time stability we present the spectra (eigenvalues) and energy amplification for our two flow configurations, plane Poiseuille and plane Couette flow (see figure 4). We note that the system matrices arising from the discretization of the linearized Navier-Stokes equations for plane Poiseuille and plane Couette flow are highly nonnormal; in fact, the degree of nonnormality increases exponentially with Reynolds number. This is confirmed by the transient energy growth $G(t)$ displayed in figure 4 . The results in the figure are ob- 


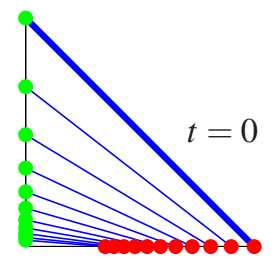

normal

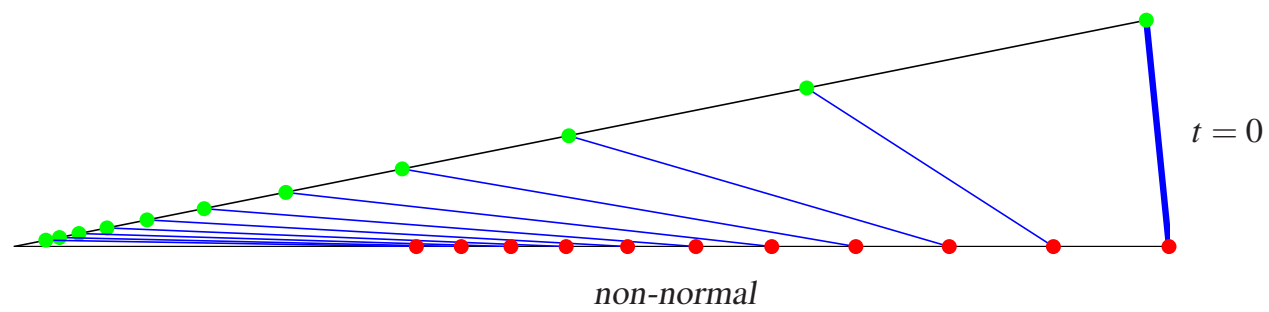

Fig. 3. Geometric interpretation of transient growth.
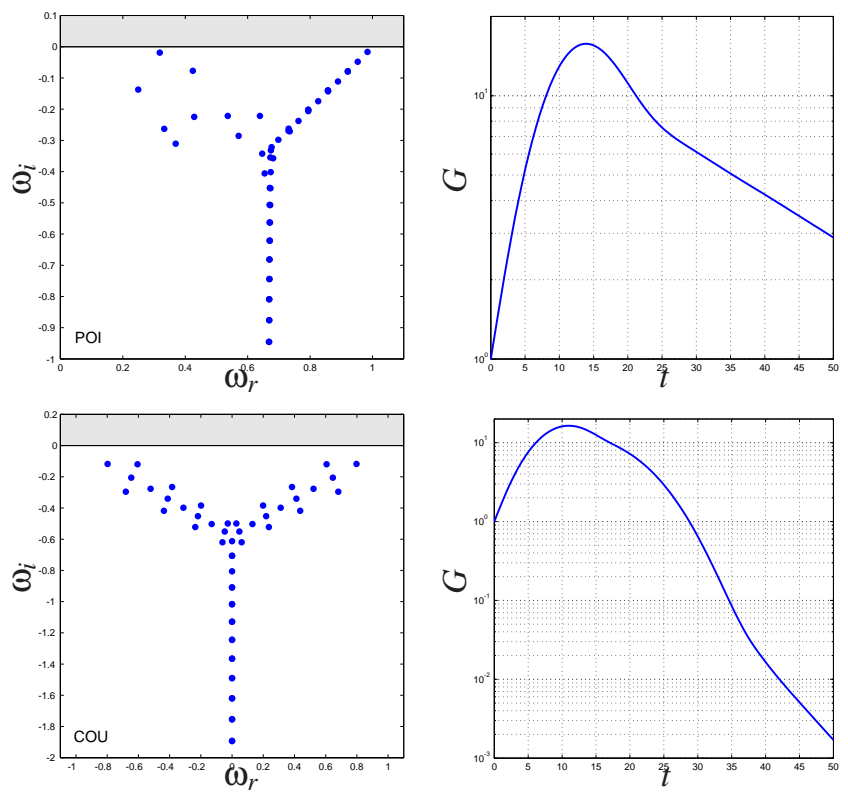

Fig. 4. Spectrum (left column) and transient energy growth (right column) for plane Poiseuille flow (top row) and plane Couette flow (bottom row). The parameters for plane Poiseuille flow are: $\alpha=$ $1, \beta=0.25, R e=2000$; the parameters for plane Couette flow are: $\alpha=1, \beta=0,25, \operatorname{Re}=1000$. Results obtained with the routine TransientGrowth.m.

tained with the routine Transient Growth.m. The choice of parameters for the two flow cases produces stable spectra: all the eigenvalues are confined to the stable half-plane. Consequently, we expected exponential energy decay according to the least stable eigenvalue for sufficiently large times. Due to their nonnormality, however, substantial energy amplification is possible before the time-asymptotic behavior sets in. The $G(t)$-curves in figure 4 show energy amplification of more than one order of magnitude over the initial energy within a time-span of $t \approx 13$.

\subsection{The numerical abscissa and the numerical range}

We learned that for nonnormal systems the eigenvalues of $L$ describe the time-asymptotic behavior of disturbances, but fail to capture the short-time dynamics. The matrix exponential captures the entire perturbation dynamics, but is costly to evaluate for many realistic applications.
A simpler tool that captures the short-time dynamics can be derived by taking advantage of a Taylor-series expansion of the matrix exponential around $t=0^{+}$, that is $\exp (t \mathrm{~L}) \approx$ $\mathrm{I}+t \mathrm{~L}+\ldots$ Starting with the definition of the energy growth rate at short time, we can readily derive

$$
\begin{aligned}
\left.\frac{d G}{d t}\right|_{t=0^{+}} & =\left.\max _{\mathbf{q}_{0}} \frac{1}{\left\|\mathbf{q}_{0}\right\|^{2}} \frac{d}{d t}\left\|(\mathrm{I}+t \mathrm{~L}) \mathbf{q}_{0}\right\|^{2}\right|_{t=0^{+}}, \\
& =\left.\max _{\mathbf{q}_{0}} \frac{d}{d t} \frac{\left\langle(\mathrm{I}+t \mathrm{~L}) \mathbf{q}_{0},(\mathrm{I}+t \mathrm{~L}) \mathbf{q}_{0}\right\rangle}{\left\langle\mathbf{q}_{0}, \mathbf{q}_{0}\right\rangle}\right|_{t=0^{+}}, \\
& =\max _{\mathbf{q}_{0}} \frac{\left\langle\mathbf{q}_{0},\left(\mathrm{~L}+\mathrm{L}^{H}\right) \mathbf{q}_{0}\right\rangle}{\left\langle\mathbf{q}_{0}, \mathbf{q}_{0}\right\rangle}, \\
& =\lambda_{\max }\left(\mathrm{L}+\mathrm{L}^{H}\right) .
\end{aligned}
$$

In this derivation, we see that the slope of the gain curve $G(t)$ at $t=0^{+}$is given by the maximum Rayleigh quotient of the composite matrix $\mathrm{L}+\mathrm{L}^{H}$. This latter matrix is Hermitian and thus normal, even though $L$ by itself may be non-normal. Consequently, the maximum Rayleigh quotient is formed by choosing the principal eigenvector of $\mathrm{L}+\mathrm{L}^{H}$ for $\mathbf{q}_{0}$. The resulting value of the Rayleigh quotient and therefore the slope of the gain curve $G(t)$ at $t=0^{+}$is given by the largest (real) eigenvalue of $\mathrm{L}+\mathrm{L}^{H}$ which is expressed in the last line of the above derivation. This quantity is referred to as the numerical abscissa of $\mathrm{L}$.

We can summarize our findings for non-normal stability problems so far as the short-time $\left(t=0^{+}\right)$dynamics is described by the eigenvalue of $\mathrm{L}+\mathrm{L}^{H}$ with the largest real part, while the long-time $(t \rightarrow \infty)$ dynamics is represented by the eigenvalue of $L$ with the largest real part.

We can learn even more by generalizing the concept of the numerical abscissa to the concept of the numerical range. To this end, we proceed by considering the energy growth rate (at any time $t$ ) and follow a similar procedure than outlined above for the numerical abscissa. We have

$$
\begin{aligned}
\gamma(t) & =\frac{1}{E} \frac{d E}{d t}=\frac{1}{\|\mathbf{q}\|_{E}^{2}} \frac{d}{d t}\langle\mathbf{q}, \mathbf{q}\rangle_{E}, \\
& =\frac{1}{\langle\mathbf{q}, \mathbf{q}\rangle_{E}}\left(\left\langle\frac{d \mathbf{q}}{d t}, \mathbf{q}\right\rangle_{E}+\left\langle\mathbf{q}, \frac{d \mathbf{q}}{d t}\right\rangle_{E}\right), \\
& =2 \operatorname{Real}\left(\frac{\langle L \mathbf{q}, \mathbf{q}\rangle_{E}}{\langle\mathbf{q}, \mathbf{q}\rangle_{E}}\right) .
\end{aligned}
$$


(a)

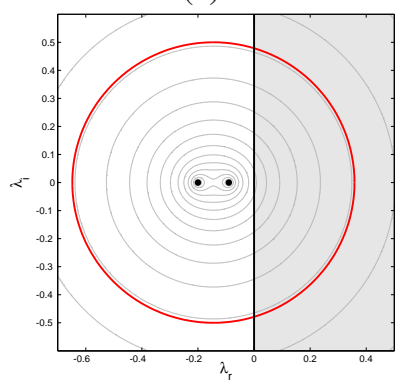

(b)

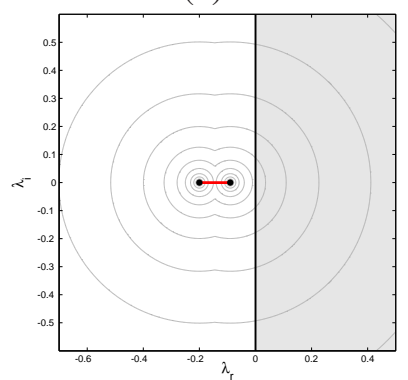

Fig. 5. Illustration of the numerical range using the $2 \times 2$ model problem for $R e=10$. (a) Choosing $\mu=1$ results in a non-normal matrix and a numerical range (delimited by the red curve) which is detached from the spectrum (black symbols); (b) for $\mu=0$ we have a normal matrix with identical eigenvalues, but a numerical range that deteriorates to the convex hull of the two eigenvalues, given simply by a line connecting the two eigenvalues.

The last expression establishes a link between the energy growth rate $\gamma(t)$ and the set of all Rayleigh quotients $\langle\operatorname{Lq}, \mathbf{q}\rangle_{E} /\langle\mathbf{q}, \mathbf{q}\rangle_{E}$. This latter set is known as the numerical range of $\mathrm{L}$ and represents a set in the complex plane.

For our purposes, three properties of the numerical range are important. First, the numerical range of $L$ is a convex set in the complex plane; a line connecting any two points in the set is entirely contained in the set. Second, the numerical range contains the spectrum of $\mathrm{L}$, which can easily verified since the Rayleigh quotient coincides with an eigenvalue of $L$ when choosing the corresponding eigenvector of $L$ as $q$ in the above expression. Third, and less obvious, the numerical range degenerates into the convex hull of the spectrum of $L$ if $L$ is normal, with the convex hull being the smallest convex set that contains the spectrum.

Again, we use our $2 \times 2$ model problem to illustrate the concept of the numerical range. We choose a Reynolds number of $R e=10$ and $\mu=1$ and plot the numerical range in the complex plane, together with the spectrum of A (see figure 5). We verify that for nonnormal matrices $A$ the numerical range is convex and contains the spectrum (the eigenvalues, illustrated by the two black symbols in figure 5(a)). We observe that the numerical range reaches into the unstable half-plane, indicated in gray. This means that there exist positive energy growth rates, despite the fact that both eigenvalues are confined to the stable half-plane. By choosing $\mu=0$ in the model problem (and thus diagonalizing the system matrix), we arrive at a normal problem. In this case, the numerical range collapses to the convex hull of the spectrum, simply given by a connecting line between the two eigenvalues. In other words, all Rayleigh quotients that can be formed with this normal matrix $\mathrm{A}$ (for $\mu=0$ ) fall on this line. In this case, the entire numerical range (the connecting line) is contained in the stable half-plane and no positive energy growth is possible. The least stable eigenvalue governs the dynamics of the system for all times.

Before discussing the numerical range for our two cases of plane Poiseuille flow and plane Couette flow, we present
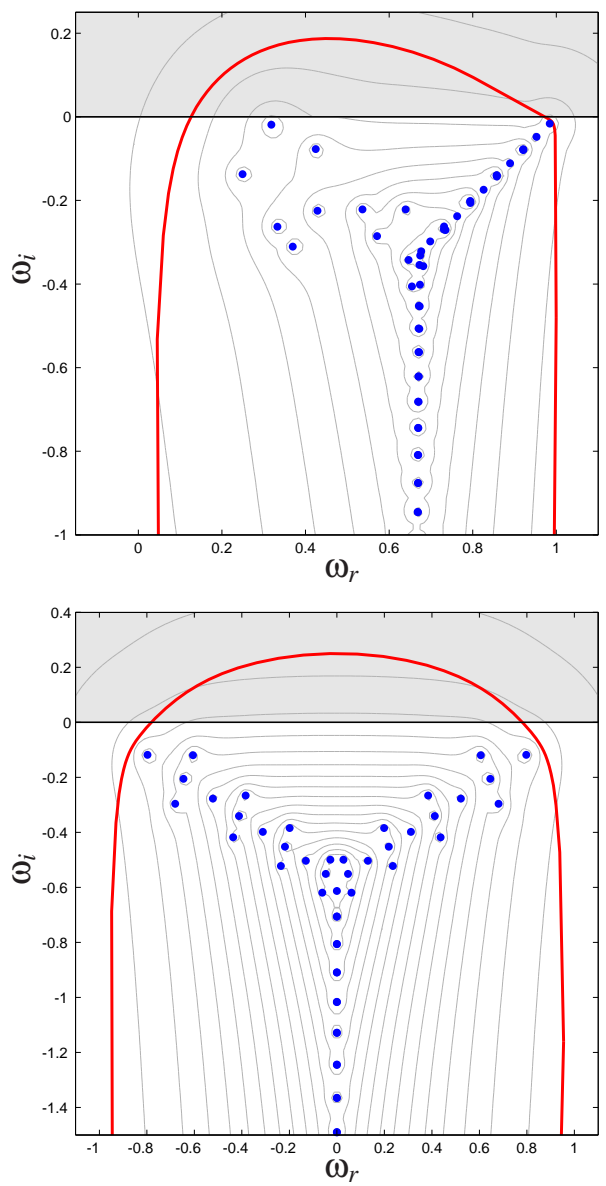

Fig. 6. Numerical range (red boundary), resolvent contours and spectrum (blue symbols) for plane Poiseuille flow (top) and plane Couette flow (bottom). The parameters are $\alpha=1, \beta=0.25$ and $R e=2000$ for plane Poiseuille flow and $R e=1000$ for plane Couette flow. The results are obtained with the routine NumRange. $\mathrm{m}$.

a numerical algorithm to compute the boundary of the numerical range [3]. This algorithm is based on the fact that the numerical abscissa, i.e., $\lambda_{\max }\left(A+A^{H}\right)$, coincides with the right-most point of the numerical range. By rotating the matrix through an angle of $2 \pi$ we can thus trace out the boundary of the numerical range by repeated numericalabscissa calculations. More specifically, we form a matrix $\mathrm{N}=\exp (i \theta) \mathrm{A}$ and its Hermitian component $\overline{\mathrm{N}}=\mathrm{N}+\mathrm{N}^{H}$. A point $z$ on the boundary of the numerical range is then given by $z(\theta)=\left(\mathbf{v}_{\max }^{H} A \mathbf{v}_{\max }\right) /\left(\mathbf{v}_{\max }^{H} \mathbf{v}_{\max }\right)$ with $\mathbf{v}_{\max }$ as the principal eigenvector (corresponding to the principal eigenvalue) of $\bar{N}$. As the angle $\theta$ traverses through the interval $[02 \pi]$, the point $z$ traces out the boundary of the numerical range.

The spectrum and numerical range for plane Poiseuille and plane Couette flow is presented in figure 6 . The results in the figure are obtained with the routine NumRange. $\mathrm{m}$. Parameters have been chosen for plane Poiseuille flow that render the parabolic mean flow asymptotically stable; plane Couette flow, on the other hand, is asymptotically stable for all Reynolds numbers. In both cases, however, we observe that the numerical range, indicated by the red contour, 


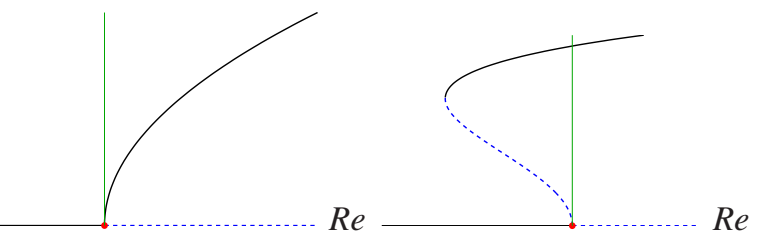

Fig. 7. Sketch of supercritical (left) and subcritical (right) bifurcation behavior. The critical parameter is indicated by the red symbol. Dashed lines denote unstable branches. For supercritical behavior, finite-amplitude states exist only after the (linear) infinitesimalamplitude state has gone unstable (right of the thin green line). For subcritical behavior, finite-amplitude states exist even before the (linear) infinitesimal-amplitude state has become unstable (left of the thin green line).

reaches far into the unstable half-plane (shaded in gray). We thus conclude that initial energy growth is possible - up to a growth rate given by the maximum protrusion of the numerical range into the unstable half-plane - but that asymptotic, exponential decay follows as time tends to infinity. The gray contour lines, indicating isolines of constant resolvent norm, will be discussed later.

\subsection{Supercritical versus subcritical bifurcation behav- ior}

For incompressible flow the two stability analysis tools, numerical range and spectrum, allow us to establish an interesting connection between non-normality and bifurcation behavior. It is easy to verify that the nonlinear terms of the incompressible Navier-Stokes equations are energypreserving: the role of the nonlinear terms is the distribution, scattering and transfer of energy, but this reorganization is accomplished in a conservative manner. Energy growth or decay can only come from linear processes. Energy growth, however, is necessary to reach finite-amplitude states. For normal systems, energy growth is only possible through unstable eigenvalues, since for normal systems the numerical range is attached to the spectrum (via the convex-hull condition) and both numerical range and spectrum cross into the unstable half-plane at the same value of the governing parameters. For this reason, finite-amplitude states can only be reached, after the infinitesimal state has become unstable. This type of bifurcation is known as supercritical (see left subplot of figure 7 for a sketch of supercritical bifurcation behavior). Rayleigh-Bénard convection, for example, falls into this category. Normal systems (with energy-conserving nonlinearities) thus reach nonlinear finite-amplitude states supercritically.

In contrast, subcritical bifurcation behavior is characterized by the existence of nonlinear, finite-amplitude states at values of the governing parameter where the infinitesimal state is still stable (left of the thin green line in figure 7b). In order to generate energy growth to reach this finite-amplitude state, we need linear energy amplification of an asymptotically stable system. In other words, the numerical range has to protrude into the unstable half-plane, when the spectrum is still confined to the stable half-plane. This configuration is only possible for a nonnormal system. Both plane Poiseuille flow and plane Couette flow fall into this category; they behave subcritically as the governing parameter (commonly the Reynolds number) is varied.

We conclude from this above argument, that normal systems behave supercritically and that subcritical bifurcation behavior necessitates a nonnormal underlying system matrix. This argument holds only if the nonlinearities cannot contribute to energy growth, that is, when energy amplification can only stem from a linear process.

\subsection{Parameter dependence}

The analysis above has been demonstrated on both a model problem and on two generic flow configurations. The governing equations for the fluid systems contain numerous parameters: the Reynolds number $R e$, the streamwise and spanwise wavenumbers $\alpha, \beta$, the time horizon $t$. It is thus natural to ask how short-time energy amplification and asymptotic behavior depend on these parameters and, in particular, which structures (given by their streamwise and spanwise dependence) optimally exploit the transient growth of energy.

For this parameter study we will trace three quantities: (i) the maximum protrusion of the numerical range into the unstable half-plane (which is negative if the numerical range is contained in the stable half-plane), (ii) the maximum transient energy amplification given as $G_{\max }=\max _{t>0} G(t)$ and (iii) the growth/decay rate of the least stable eigenvalue.

First, we will set $\beta=0$ and consider only twodimensional waves propagating in the streamwise direction. We then vary the remaining parameters $\alpha$ and $R e$ and determine the maximum energy growth $G_{\max }$, the numerical abscissa and the growth rate of the least stable eigenvalue, thus covering the short-time (numerical abscissa), intermediatetime $\left(G_{\max }\right)$ and long-time (least stable eigenvalue) behavior of the flow. The results of these computations are shown in figure 8 for plane Poiseuille flow (left) and plane Couette flow (right). For Poiseuille flow we observe three domains delimited by the zero-contour of the numerical abscissa (white contour line) and the zero-contour of the growth rate of the least stable eigenvalue, resulting in the parameter space where exponential instabilities exist (gray area). The latter contour is rather familiar and referred to as the neutral curve for plane Poiseuille flow. Its left-most point determines the critical Reynolds number of $R e=5772$, i.e. the smallest Reynolds number above which infinitesimal perturbations will show asymptotic exponential growth. This growth is realized by streamwise waves $(\beta=0)$ with a wavelength of about $\alpha=1.02$. To the left of the zero-contour of the numerical abscissa the flow exhibits monotonic energy decay. The parameter range enclosed between these two zero-contours are characterized by transient growth followed by exponential decay. The same calculations for plane Couette flow are qualitatively different in as far as this type of flow is asymptotically stable for all parameter combination and thus does not have a neutral curve. Nevertheless, a substantial amount of transient energy growth can be observed above the zero- 

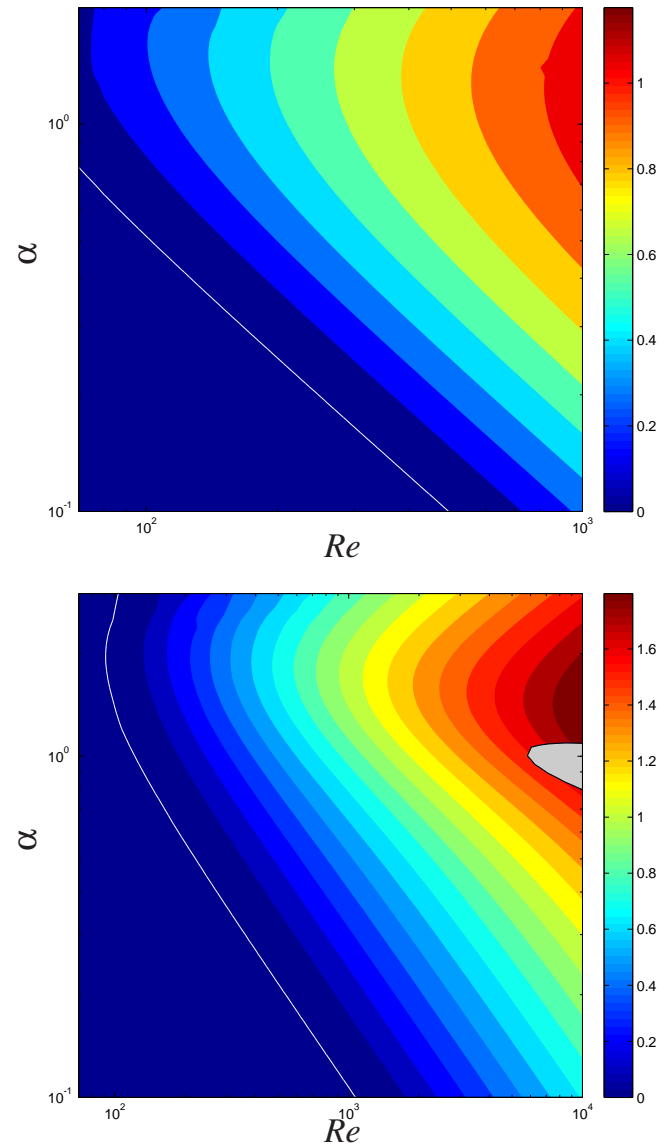

Fig. 8. Parametric study of maximum transient growth as a function of the streamwise wavenumber and Reynolds number $(\alpha, R e)$ for plane Poiseuille flow (top) and plane Couette flow (bottom). The spanwise wavenumber in both cases is $\beta=0$. The area shaded in gray (for plane Poiseuille flow) denotes the parameter space for exponential (modal) growth. The white contour line is given by a zero value of the numerical abscissa. The contour levels represent $\log _{10}\left(G_{\max }\right)$. The results are obtained with the routine Neutral_a_Re.m.

contour of the numerical abscissa.

In a second parameter study, we investigate the transient growth potential for a fixed Reynolds number but varying wavenumbers. This is equivalent of asking which waves are most favored by the transient energy amplification mechanism. For asymptotic long-time considerations, Squire's theorem states that for every unstable three-dimensional perturbation there exists a two-dimensional $(\beta=0)$ unstable perturbation at a lower Reynolds number. For this reason, it suffices to compute the asymptotic growth-rates of twodimensional waves (with $\beta=0$ ) when determining the longtime behavior for plane Poiseuille flow. Squire's theorem does not hold for transient growth or short-time instabilities, however, and figure 9 shows the result of $G_{\max }$-calculations for varying wavenumbers $\alpha$ and $\beta$. For plane Poiseuille flow, we have chosen a Reynolds number of $R e=10000$, above the critical ones; consequently, a region (in gray) where infinite energy amplification can be obtained due to an ex-
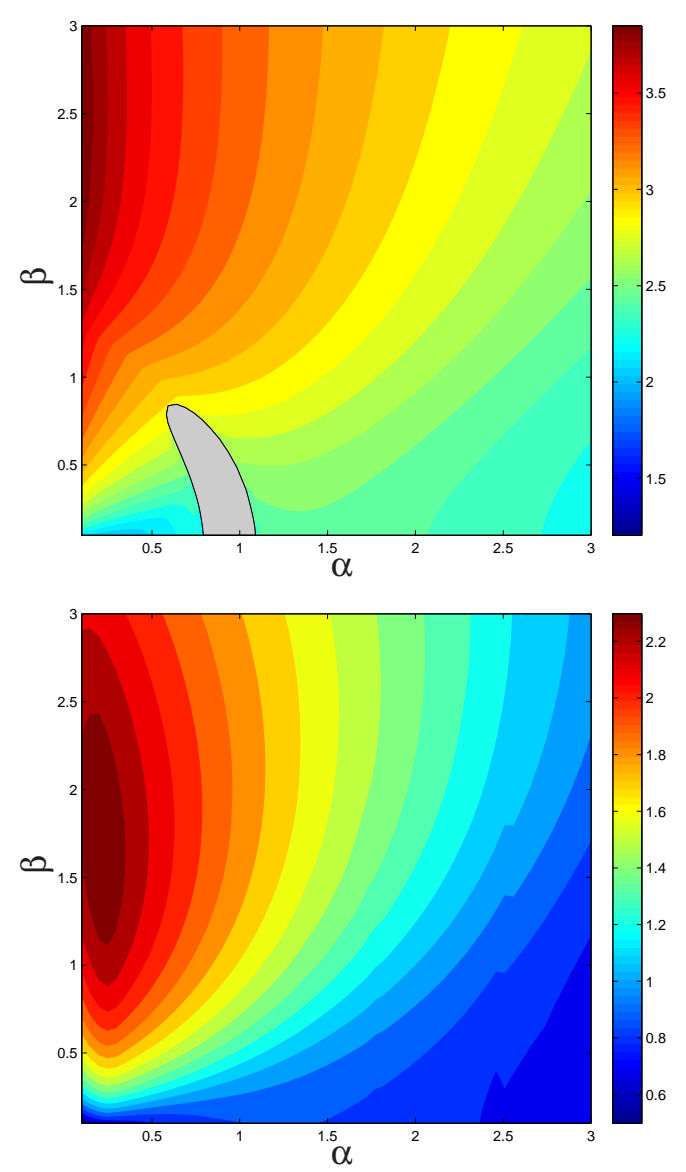

Fig. 9. Parametric study of maximum transient growth as a function of the streamwise and spanwise wavenumber $(\alpha, \beta)$ for plane Poiseuille flow (top, $R e=10000$ ) and plane Couette flow (bottom, $R e=500$ ). The area shaded in gray (for plane Poiseuille flow) denotes the parameter space for exponential (modal) growth. The contour levels represent $\log _{10}\left(G_{\max }\right)$. The results are obtained with the routine Neutral_alpha_beta.m.

ponential instability is include in the figure. Squire's theorem is confirmed as two-dimensional waves (with $\beta=0$ ) are most favored by the exponential, eigenvalue-based instability. A different picture emerges for transiently amplified waves: perturbations that show no streamwise dependence $(\alpha=0)$ are most amplified. The maximum occurs at a spanwise wavenumber of about $\beta=2$. A similar behavior can be observed for plane Couette flow (see figure 9(bottom); $R e=1000)$. The most amplified waves can be found near the $\beta$-axis for $\beta \approx 2$. In contrast to plane Poiseuille flow, the maximum is reached for a non-zero, but small streamwise wavenumber.

For our two fluid configurations - and in general for nonnormal system - we can distinguish three genuine regimes of flow behavior parameterized by the governing parameter, in our case the Reynolds number. These regimes are given by the critical Reynolds number at which either the numerical range or the spectrum cross into the unstable halfplane. In the first regime, both numerical range and spectrum are contained in the stable half-plane and we observe 


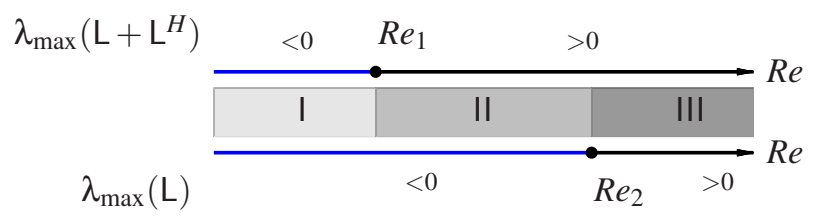

Fig. 10. Three parameter domains of qualitative stability behavior: in region I $\left(R e<R e_{1}\right)$ both the numerical abscissa and the growth rate of the least stable eigenvalue are negative, resulting in monotonic energy decay; in region II $\left(R e_{1}<R e<R e_{2}\right)$ the numerical abscissa is positive, but the eigenvalues are still confined to the stable half-plane, yielding short-time energy amplification followed by asymptotic decay; in region III $\left(R e>R e_{2}\right)$ both the numerical range and the spectrum have crossed into the unstable half-plane, giving rise to transient and asymptotic energy growth.

Table 1. Table of critical Reynolds numbers for the numerical abscissa $\left(R e_{1}\right)$ and the spectrum $\left(R e_{2}\right)$ for our model problem, our two flow cases and thermal convection. [nn: nonnormal system matrix, n: normal system matrix.]

\begin{tabular}{lllll}
\hline case & type & $\mathrm{L}$ & $\operatorname{Re}_{1}$ & $\operatorname{Re}_{2}$ \\
\hline $2 \times 2$ model $(\mu=1)$ & $\mathrm{nn}$ & $\mathrm{A}$ & $\sqrt{8}$ & 100 \\
Poiseuille flow & $\mathrm{nn}$ & $\mathrm{L}_{p o i}$ & 49.6 & 5772.2 \\
Couette flow & $\mathrm{nn}$ & $\mathrm{L}_{c o u}$ & 20.7 & $\infty$ \\
thermal convection & $\mathrm{n}$ & $\mathrm{L}_{t c}$ & 1705 & 1705 \\
\hline
\end{tabular}

monotonic energy decay. The second regime is characterize by a numerical range protruding into the unstable halfplane and the entire spectrum in the stable half-plane; in this case, we can observe short-term energy growth followed by asymptotic decay. Finally, the third regime is determined by both numerical range and at least one eigenvalue in the unstable half-plane; in this regime, we exhibit asymptotic, exponential growth, which may still be preceded by significant transient growth before the time-asymptotic behavior prevails. Figure 10 sketches these three regimes, while table 1 lists the critical Reynolds numbers for plane Couette, plane Poiseuille, Rayleigh-Bénard convection (a normal system) and our simple $2 \times 2$ model problem. While for nonnormal systems the two critical Reynolds numbers for the crossing of the numerical range and spectrum are vastly different, they coincide for the normal Rayleigh-Bénard system since in this case the numerical range is the convex hull of the spectrum and crosses with the spectrum into the unstable half-plane.

\subsection{How to recover the optimal initial condition}

For non-normal systems that exhibit substantial transient growth, it is often instructive to calculate the specific initial condition that reaches maximum energy amplification $G_{\text {max }}$ or an energy amplification at another user-specified
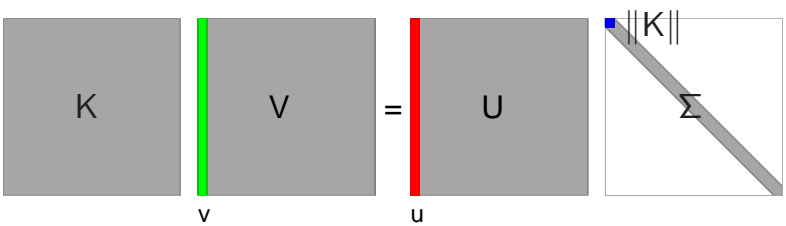

Fig. 11. Sketch of the computational procedure to recover the optimal initial condition via a singular value decomposition.

time $t^{*}$. Recalling the definition of the matrix exponential norm, it is given by an optimization over all initial conditions; the resulting curve $G(t)$ is thus an envelope over many individual realizations. In fact, each point on the curve $G(t)$ may have been generated by a different initial condition. The initial condition that yields optimal energy amplification at a given time $t^{*}$ we write

$$
\exp \left(t^{*} \mathrm{~L}\right) \mathbf{q}_{0}=\left\|\exp \left(t^{*} \mathrm{~L}\right)\right\| \mathbf{q}
$$

where the unit-norm initial condition $\mathbf{q}_{0}$ is advanced in time by $\exp \left(t^{*} \mathrm{~L}\right)$ producing the unit-norm state vector $\mathbf{q}$ that is amplified by $G\left(t^{*}\right)=\left\|\exp \left(t^{*} \mathrm{~L}\right)\right\|$. The above expression is reminiscent of a singular value decomposition (SVD), or $\mathrm{KV}=\mathrm{U} \Sigma$ where $\mathrm{V}$ and $\mathrm{U}$ are unitary matrices with orthonormalized columns and $\Sigma$ is a diagonal matrix containing the singular values. Recalling that the dominant singular value is equivalent to the $L_{2}$-norm of $\mathrm{K}$ we can extract the principal components from the singular value decomposition and write

$$
\mathrm{K} \mathbf{v}_{1}=\|\mathrm{K}\| \mathbf{u}_{1}
$$

where the index ${ }_{1}$ denotes the first column of $\mathrm{V}$ or $\mathrm{U}$ (see figure 11). Matching this last expression to (20), it is straightforward to identify the optimal initial condition $\mathbf{q}_{0}$ as the principal right singular vector of $\exp \left(t^{*} \mathrm{~L}\right)$ and the resulting state-vector $\mathbf{q}$ as the principal left singular vector. The computational steps to recover the optimal initial condition thus simply involve a singular value decomposition of the matrix exponential evaluated at the user-specified time $t^{*}$. The routine OptimalDisturbance. m computes the optimal initial condition and the corresponding flow response, the output.

\section{Receptivity analysis of fluid systems}

The revised role of eigenvalues for describing the behavior of non-normal systems, see also [4], carries over to many other areas where eigenvalues have traditionally dominated. One such area is receptivity analysis which is concerned with the general response of a fluid system to external disturbances. These external disturbances can take on the shape of free-stream turbulence, acoustic perturbations or 
wall roughness, among many other possibilities. The maximum response in energy of the fluid system to a unit-energy forcing is a reasonable and common receptivity measure. Often, receptivity is described via a resonance argument, given by the closeness of the external frequencies to any of the eigenvalues of the driven system. As we will see below, this argument is valid and accurate for normal systems. For non-normal systems, however, this eigenvalue-based analysis proves inadequate.

\subsection{The resolvent norm}

We will return to our general fluid system and include the driving term introduced earlier. In addition, we adopt an input-output framework and introduce a supplementary equation that evaluates a user-specified component $\mathbf{g}$ of the full state-vector $\mathbf{q}$. Within this framework, the matrices B and $\mathrm{C}$ determine the input and output quantities, respectively. We have

$$
\begin{aligned}
\frac{d}{d t} \mathbf{q} & =\mathrm{L} \mathbf{q}+\mathrm{B} \mathbf{f} \\
\mathbf{g} & =\mathrm{C} \mathbf{q} .
\end{aligned}
$$

The above linear equation can readily be solved yielding the expression

$$
\mathbf{g}(t)=\int_{0}^{t} \mathrm{C} \exp ((t-\tau) \mathrm{L}) \mathrm{B} \mathbf{f}(\tau) d \tau
$$

which constitutes a memory integral where the current output state $\mathbf{g}$ depends on the entire history of the forcing $\mathbf{f}$. In the above expression we assumed a zero initial condition, $\mathbf{q}_{0}=0$. For stable systems $L$ the influence of the forcing on the current state decays exponentially according to the decay rate of the least stable eigenvalue. Even though the above equation could be solved numerically, we will make a further assumption regarding the form of the forcing and assume a harmonic external driving $\mathbf{f}=\hat{\mathbf{f}} \exp (i \omega t)$. Due to the linearity of the governing equations, the output $\mathbf{g}$ responds with the same frequency and can also be represented as $\mathbf{g}=\hat{\mathbf{g}} \exp (i \omega t)$. Furthermore, the above memory integral simplifies to

$$
\hat{\mathbf{g}}=\mathrm{C}(i \omega \mathbf{l}-\mathrm{L})^{-1} \mathrm{~B} \hat{\mathbf{f}}
$$

which presents a mapping between the harmonic input forcing and the corresponding output response. Analogous to the case treated in section $\S 3$, we define the maximum gain in energy by harmonic forcing as the ratio of driving energy to response energy, maximized over all possible forcing profiles $\hat{\mathbf{f}}$, but for a given forcing frequency $\omega$. We obtain

$$
\begin{aligned}
R(\omega) & =\max _{\hat{\mathbf{f}}} \frac{\|\hat{\mathbf{g}}\|_{E}^{2}}{\|\hat{\mathbf{f}}\|_{E}^{2}}, \\
& =\max _{\hat{\mathbf{f}}} \frac{\left\|\mathrm{C}(i \omega \mathbf{l}-\mathrm{L})^{-1} \mathrm{~B} \hat{\mathbf{f}}\right\|_{E}^{2}}{\|\hat{\mathbf{f}}\|_{E}^{2}}, \\
& =\left\|\mathrm{C}(i \omega \mathbf{l}-\mathrm{L})^{-1} \mathrm{~B}\right\|_{E}^{2} .
\end{aligned}
$$

The final expression is referred to as the resolvent norm, measuring the maximum response due to harmonic forcing, optimized over all forcings.

By changing from an initial-value problem to a harmonically driven problem, we replace the matrix exponential norm with the resolvent norm to quantify the amplification of energy in our system. We also notice that the resolvent can be related to the matrix exponential via a Laplace transform.

For plane Poiseuille and plane Couette flow, the resolvent norm is shown in figure 12 as a function of forcing frequency $\omega$. The results are obtained with the routine Resolvent.m which also displays the resolvent norm in the complex $\omega$-plane. We detect strong peaks, indicating a strong response to forcing at the peak frequencies. These strong peaks appear correlated to the location of the least stable eigenvalues of the respective flows. Alternatively, these plots can also be thought of as transfer functions where the system given by $L$ acts as a filter: amplifying certain frequencies while damping others.

\subsection{The resonant limit}

The resolvent norm is a less familiar concepts for quantifying forced responses to external, harmonic driving, just as the matrix exponential norm is less common than an assessment of the spectrum for stability considerations. As before, we apply an eigenvalue decomposition of the system matrix, i.e., $\mathrm{L}=\mathrm{V} \wedge \mathrm{V}^{-1}$, to establish a link between the resolvent norm and more standard tools for the treatment of forced solutions. We have

$$
\begin{aligned}
R(\omega) & =\left\|\mathrm{C}(i \omega \mathrm{l}-\mathrm{L})^{-1} \mathrm{~B}\right\|_{E}^{2} \\
& =\left\|\mathrm{CV}^{-1}(i \omega \mathrm{l}-\Lambda)^{-1} \mathrm{VB}\right\|_{E}^{2}
\end{aligned}
$$

The inner part of the final expression, containing the eigenvalue matrix $\Lambda$, can be written as a diagonal matrix with $1 /\left(i \omega-\lambda_{j}\right)$ on the diagonal. Each individual term measures the inverse distance of the external forcing frequency with the eigenvalues of our linear system. This is the classical definition of a resonance: the coincidence of the driving frequency with an eigenfrequency of the driven system. This classical definition of a resonance (based on eigenvalues only) discards the information contained in the eigenvector structure the same way as the definition of stability based on the spectrum ignored the same information. For normal system matrices $L$ this is justified as the eigenvector matrix 

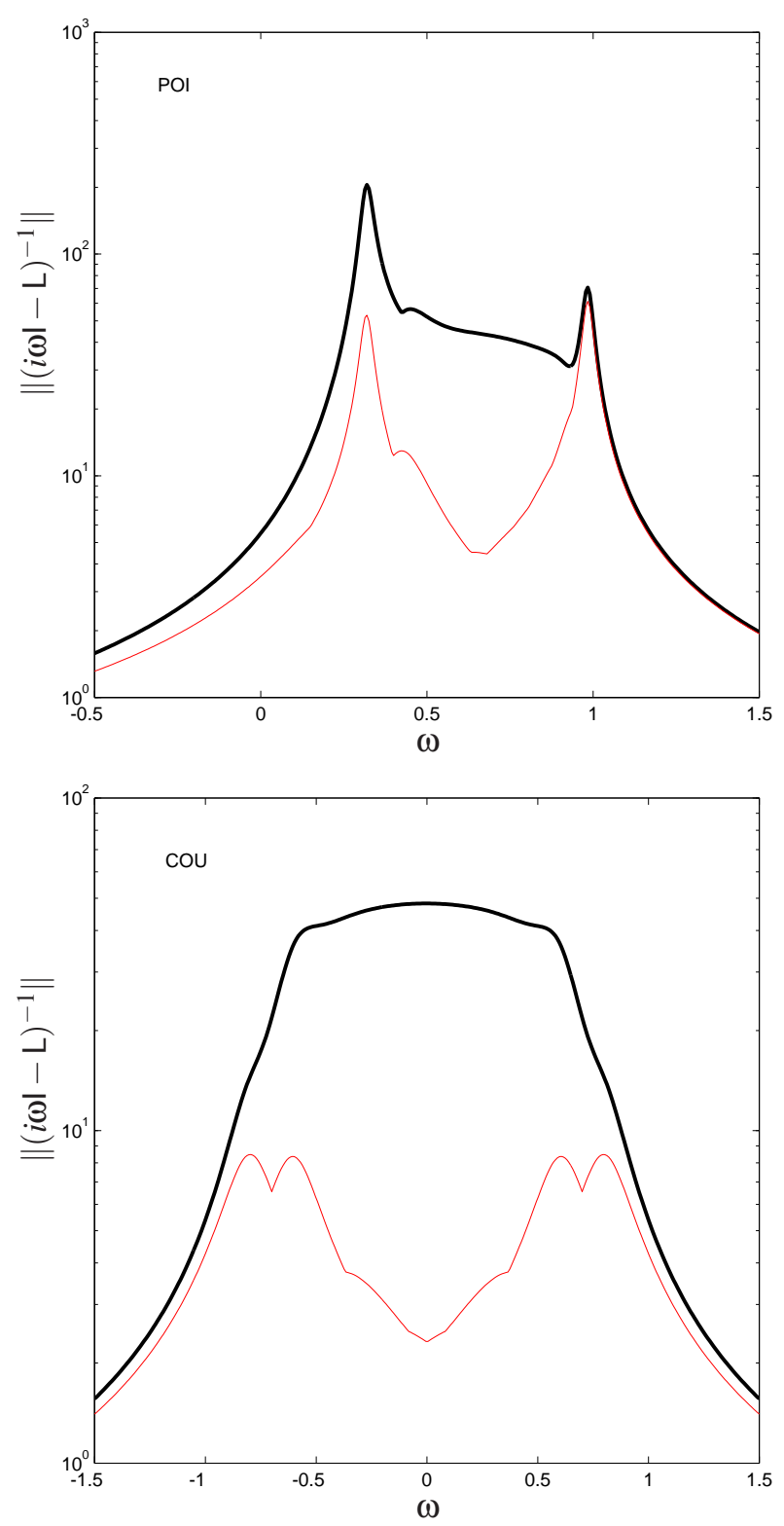

Fig. 12. Resolvent norm $\left\|(i \omega \mathrm{l}-\mathrm{L})^{-1}\right\|$ for plane Poiseuille (top) and plane Couette flow (bottom), thick black line. The parameters are: $\alpha=1, \beta=0.25$ and $R e=2000$ for plane Poiseuille flow and $R e=1000$ for plane Couette flow. The thin red line represents the resonant limit, based on the inverse of the minimal distance of the forcing frequency $\omega$ to the spectrum. The results are obtained with the routine Resolvent.m.

$\mathrm{V}$ (and its inverse) is unitary in this case, representing rotations that do not alter the norm of the matrix $(i \omega \mathrm{l}-\mathrm{L})^{-1}$ in the expression above. For nonnormal system matrices $L$, though, the eigenvector structure plays an important role, and large responses due to forcing can occur even if the forcing frequency is far from an eigenvalue of the system matrix L. These instances are referred to as pseudo-resonances [4]. For highly nonnormal matrices they cannot be distinguished from true resonances. The response curves based on eigenvalues only is given by thin red lines in figure 12 ; the differ- ence between the red and black curves has to be attributed to nonnormal effects involving the non-orthogonality of the eigenvectors.

\subsection{Recovering the optimal forcing and response}

Analogous to the initial-value problem discussed in $\S 3$, it is often instructive to identify the shape of the forcing which produces the largest response in the flow, together with the flow response. To this end, we select a specific frequency $\omega^{*}$ and use, as before, the singular value decomposition $(\mathrm{SVD})$ of the matrix $\left(i \omega^{*} \mid-\mathrm{L}\right)^{-1}$. We have

$$
\left(i \omega^{*} \mathrm{I}-\mathrm{L}\right)^{-1} \mathrm{~V}=\mathrm{U} \Sigma
$$

where $\mathrm{V}$ and $\mathrm{U}$ are unitary matrices with ortho-normalized columns and $\Sigma$ is a diagonal matrix containing the singular values.

As mentioned above for the optimal initial condition, the largest singular value is equivalent to the norm of the decomposed matrix, i.e., the resolvent, and the first column of $\mathrm{V}$ and $\mathrm{U}$ define the optimal forcing and response, respectively. The computation of the optimal forcing thus amounts to a singular value decomposition of the resolvent matrix for a given forcing frequency $\omega^{*}$; see also section 3.6 and figure 11 .

Finally, we would like to point out the close link between the tools used for stability and receptivity analyses introduced in the above two sections. In both cases, we consider inputs (the initial condition $\mathbf{q}_{0}$ or the harmonic forcing $\hat{\mathbf{f}}$ ) and measure outputs (the flow at time $\mathrm{t}, \mathbf{q}(t)$, or the response $\hat{\mathbf{g}}$ ) — with a transfer matrix (the matrix exponential $\exp (t \mathrm{~L})$ or the resolvent matrix $\left.(i \omega \mathrm{l}-\mathrm{L})^{-1}\right)$ connecting the two. This connection recasts either problem as an inputoutput problem; the associated analysis is referred to as an input-output analysis.

\subsection{Input-output analysis}

The resolvent analysis based on $(i \omega \mathrm{l}-\mathrm{L})^{-1}$ measures the response of the entire state (measured by its energy) to a forcing in all components (again, measured by its energy). More information about a fluid system can be gained by being more specific about the type of forcings and the type of response. For this purpose, the matrices B and C, controlling the type of input and output, respectively, can be adjusted to determine the transfer behavior of specific forcings to specific responses. This type of analysis, referred to as componentwise input-output analysis [5], will give insight into particular input-output combinations that are specially amplified (or suppressed) by the fluid system and will allow a more mechanistic viewpoint than a pure global energy-based analysis.

Our fluid system has been formulated in a compact notation using the normal velocity and normal vorticity. For our input-output analysis, we will revert back to the three velocity component and consider the nine combinations arising from forcing by and from measuring three different velocity components. The mappings between the $\mathbf{v}, \eta$-formulation and the $\mathbf{u}, \mathbf{v}, \mathbf{w}$-formulation are given as follows 


$$
\mathbf{q}_{i n}=\underbrace{\left(\begin{array}{ccc}
i \alpha \mathrm{M}^{-1} \mathrm{D} & \mathrm{M}^{-1} k^{2} & i \beta \mathrm{M}^{-1} \mathrm{D} \\
i \beta & 0 & -i \alpha
\end{array}\right)}_{\mathrm{B}}\left(\begin{array}{c}
\mathbf{u}_{i n} \\
\mathbf{v}_{i n} \\
\mathbf{w}_{i n}
\end{array}\right),
$$

$$
\left(\begin{array}{c}
\mathbf{u}_{\text {out }} \\
\mathbf{v}_{\text {out }} \\
\mathbf{w}_{\text {out }}
\end{array}\right)=\underbrace{\left(\begin{array}{cc}
\frac{i \alpha}{k^{2}} \mathrm{D} & -\frac{i \beta}{k^{2}} \\
1 & 0 \\
\frac{i \beta}{k^{2}} \mathrm{D} & \frac{i \alpha}{k^{2}}
\end{array}\right)}_{\mathrm{C}} \mathbf{q}_{\text {out }} .
$$

The matrices $B$ and $C$ have already been introduced in the definition of the resolvent norm. We will now use their block-components to determine the transfer of energy between various velocity components in the forcing and various velocity components in the response. By considering only certain blocks and setting the remainin block to zero, we can determine the energy transfer between, say, the input normal velocity $v$ and the output velocity $u$. To eliminate the dependence on the forcing frequency, we consider the maximal response over a given frequency range. Figure 13 shows the nine combinations of input-output transfer functions for a fixed Reynolds number but for varying wavenumbers $\alpha$ and $\beta$. The colormap is constant across all panels, allowing a direct comparison. It becomes immediately obvious that the transfer from $(v, w)$ to $u$ is particularly efficient, showing the largest amplification. All other panels are far inferior in their amplification of forcing energy. In addition, for the dominant energy transfer, perturbations with a vanishing streamwise dependence constitute the preferred structures; see the black symbols indicating the maximum in each panel.

A similar picture emerges for plane Couette flow (see figure 14). Also in this case, the most efficient amplification of forcing energy follows the $v \rightarrow u$ and $w \rightarrow u$ route. And again, disturbances that are streamwise independent dominate over other structures. The most amplified waves have a spanwise wavenumber of $\beta \approx 2$. The efficient transfer of streamwise independent $(v, w)$-structures into streamwise independent $u$-structures can be attributed to the lift-up mechanism which converts streamwise vortices into streaks (streamwise indpendent $u$-perturbations) in the presence of mean shear.

The above input-output analysis, tuning the matrices $B$ and $C$, can also be used to extract physical mechanisms in complex flows. An example is provided by results reported in Klinkenberg et al. [6], based on a model by Saffman [7], showing that transient growth is enhanced when coarse dust is present in a channel flow.

We close this section by mentioning that the inputoutput formulation of linear fluid systems is both flexible and powerful and gives great insight into dominant mechanisms at play and the coherent structures that are responsible for the bulk of the energy transfer.

\section{Sensitivity analysis of fluid systems}

So far, we have studied the optimal response to initial conditions and to external forcing using an optimization point-of-view intrinsic in the matrix norm of the matrix exponential, resolvent or input-output transfer function. A related, and in a sense, more encompassing issue is the sensitivity of fluid systems to external or internal changes. The external part has already been addressed above, but will nonetheless be revisited here in light of sensitivity measures. Sensitivity analysis is the starting point for many other fluid problems, among them shape optimization, actuator/sensor placement, flow manipulations and feedback control.

The core of this section will introduce a variational framework which casts a constrained optimization problem into an unconstrained one by using adjoint variables (or Lagrange multipliers). These adjoint variables will carry sensitivity information that is valuable in its own right as well as in combination with other flow variables. The full framework is versatile and capable of answering many questions, such as: how does drag respond to periodic forcing? how does wall roughness influence dissipation rate? how do blowing/suction strategies affect mixing efficiency? how do changes in Reynolds number cause shifts in growth rates and frequencies?

\subsection{Eigenvalue sensitivity as a first indicator of nonnor- mality}

A first instructive exercise is the simple perturbation of our system matrix $L$ by small random perturbations. We are in particular interested in shifts in eigenvalues due to an additive perturbation. A simple perturbation analysis of the eigenvalue problem $\lambda \mathbf{q}=L \mathbf{q}$ can be cast into the form

$$
(\lambda+\Delta \lambda)(\mathbf{q}+\Delta \mathbf{q})=(\mathrm{L}+\Delta \mathrm{L})(\mathbf{q}+\Delta \mathbf{q})
$$

with $\Delta \mathrm{L}$ as the given matrix perturbation and $\Delta \lambda$ and $\Delta \mathbf{q}$ as the resulting perturbation in the eigenvalue and eigenvector, respectively. Rearranging the above equation and leftmultiplying with a (yet) unknown vector $\mathbf{p}$ yields

$$
\mathbf{p}^{H}(\mathrm{~L}-\lambda \mathrm{I}) \Delta \mathbf{q}=\mathbf{p}^{H}(\Delta \mathrm{L}-\Delta \lambda \mathrm{I}) \mathbf{q} .
$$

We require the left expression to be identically zero for all perturbations $\Delta \mathbf{q}$ which leads to an equation for $\mathbf{p}$ of the form

$$
\begin{aligned}
0 & =\mathbf{p}^{H}(\mathrm{~L}-\lambda \mathrm{I}), \\
& =\left(\mathrm{L}^{H}-\lambda^{*} \mathrm{I}\right) \mathbf{p} .
\end{aligned}
$$



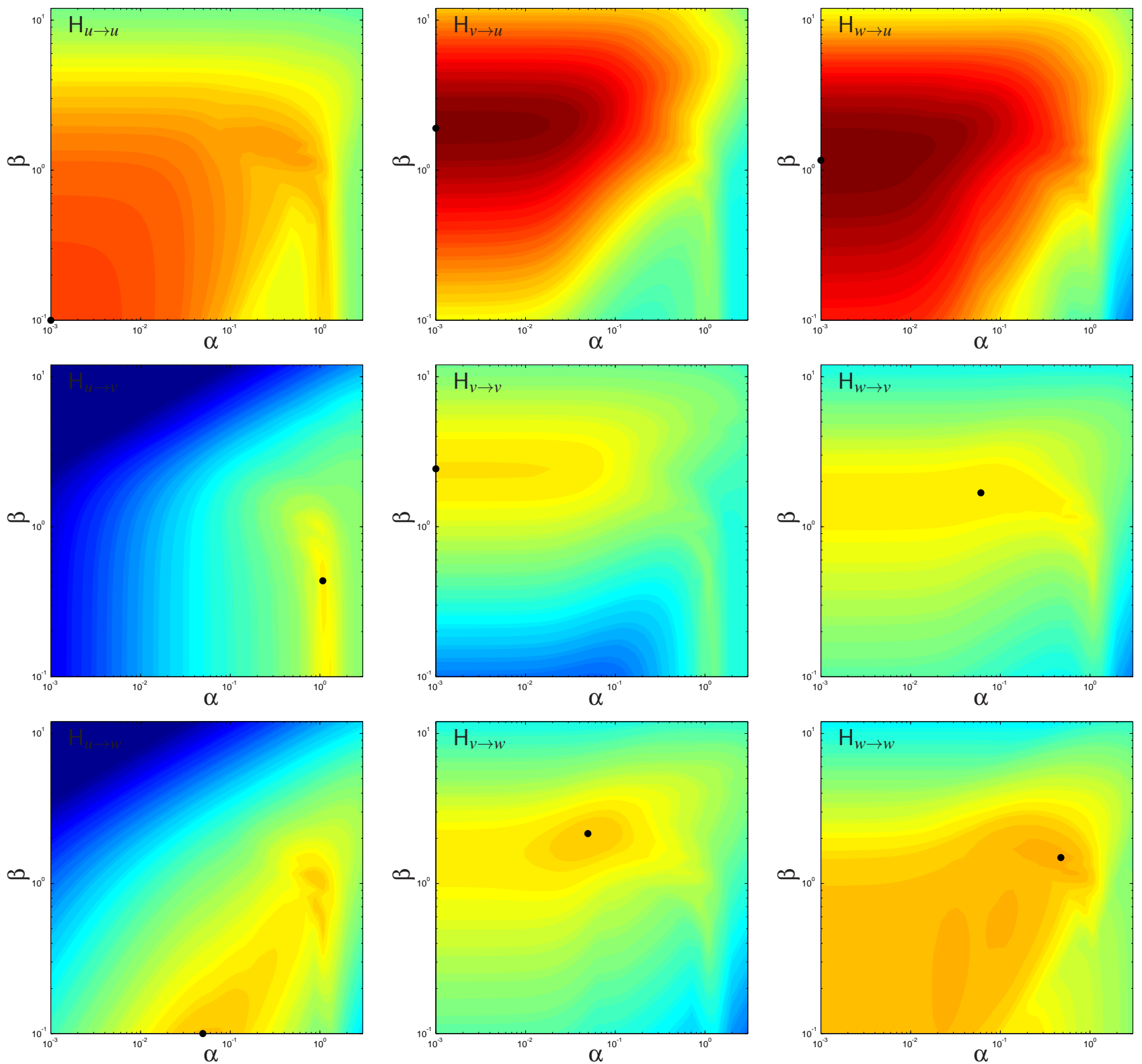

Fig. 13. Componentwise input-output analysis for plane Poiseuille flow $(R e=2000)$. Each panel displays the maximal amplification over all forcing frequencies, as a function of the streamwise and spanwise wavenumbers. In each panel, the black symbol indicates the maximum response.

The above expression identifies $\mathbf{p}$ as an eigenvector of $\mathrm{L}^{H}$, the matrix adjoint to $L$. This vector is also referred to as the adjoint or left eigenvector of $\mathrm{L}$, and the problem involving $\mathrm{L}^{H}$ is known as the adjoint problem. The eigenvalues of the adjoint problem are simply the complex conjugate of the spectrum of L. We continue with the above derivation and arrive at a relation between a matrix perturbation and the resulting eigenvalue shift of the form

$$
\Delta \lambda=\frac{\mathbf{p}^{H} \Delta \mathrm{L} \mathbf{q}}{\mathbf{p}^{H} \mathbf{q}}=\frac{\langle\mathbf{p}, \Delta \mathrm{L} \mathbf{q}\rangle}{\langle\mathbf{p}, \mathbf{q}\rangle}
$$

Bounding the response of an eigenvalue due to an additive perturbation of the matrix entries produces

$$
|\Delta \lambda| \leq \frac{\|\mathbf{p}\|\|\mathbf{q}\|}{|\langle\mathbf{p}, \mathbf{q}\rangle|}\|\Delta \mathrm{L}\|=\frac{1}{|\cos (\theta)|}\|\Delta \mathrm{L}\|
$$

where the angle $\theta$ between the direct and adjoint eigenvector appears as a proportionality constant between the norm of the matrix perturbation and the response in the associated eigenvalue.

The results of a simple numerical exercise by which the system matrix $A$ of our simple $2 \times 2$-system is perturbed by random matrices of norm $10^{-2}$ is shown in figure 15 . In 

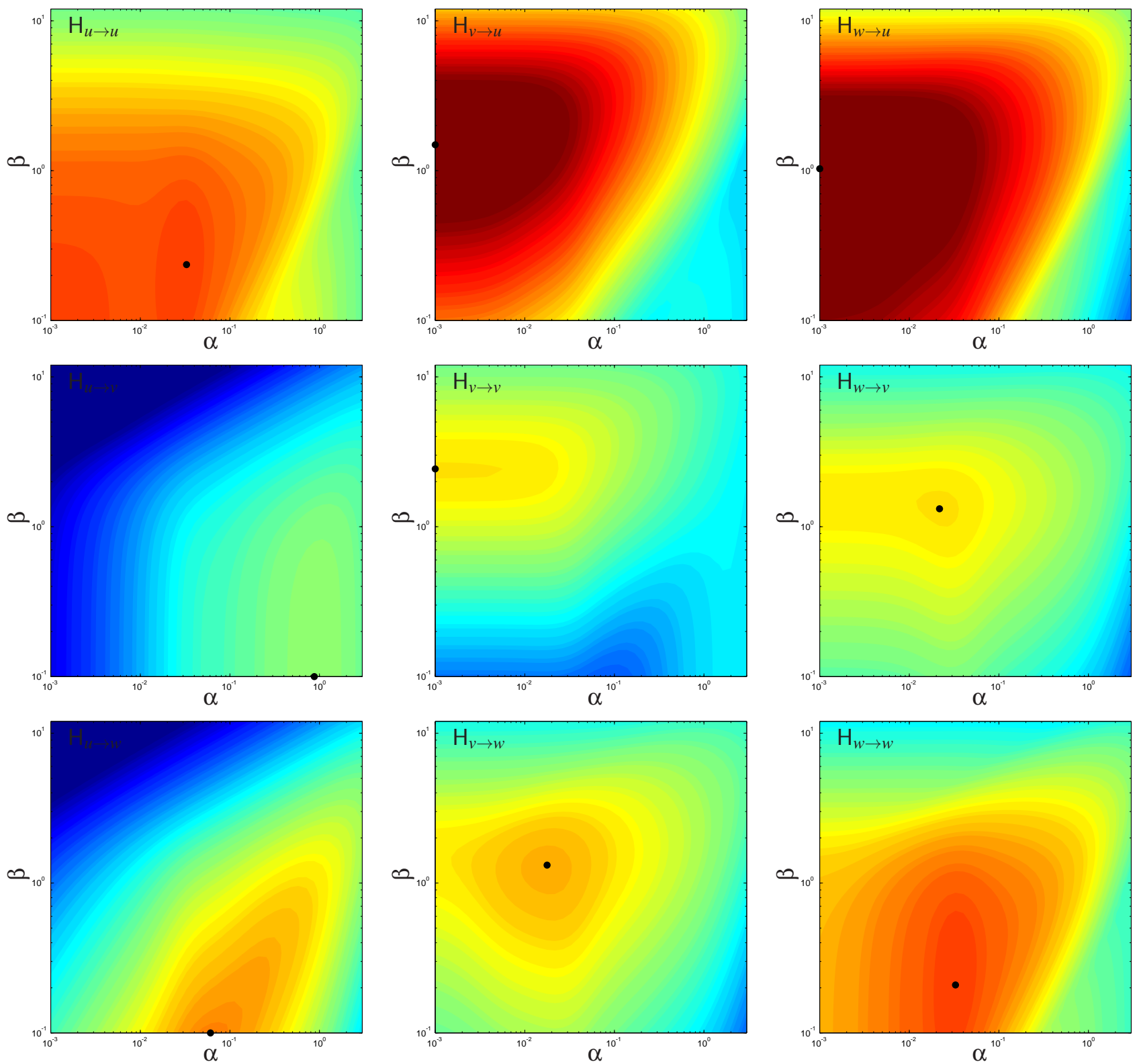

Fig. 14. Componentwise input-output analysis for plane Couette flow $(R e=1000)$. Each panel displays the maximal amplification over all forcing frequencies, as a function of the streamwise and spanwise wavenumbers. In each panel, the black symbol indicates the maximum response.

the nonnormal case $(\mu=1)$ the eigenvalues deviate by far more than $10^{-2}$ from their unperturbed location, while in the normal case $(\mu=0)$ we observe a dislocation of the perturbed eigenvalues of approximately $10^{-2}$.

The same exercise - perturbation of the stability matrix by a random matrix of norm $\varepsilon$ - can also be applied to the stability matrices of our two flow cases, plane Poiseuille and plane Couette flow. The results of this exercise is displayed in figure 16, where a superposition of the spectra of $L+\Delta L$ are shown. For a perturbation $\Delta \mathrm{L}$ of norm $\varepsilon=5 \cdot 10^{-3}$ and $\varepsilon=10^{-3}$, respectively, we see that in both cases some of the eigenvalues move by an order-one magnitude from their unperturbed locations, while other eigenvalues show very little sensitivity to the added perturbations. Also in this case, the angle between the direct and adjoint eigenvectors determines the sensitivity of the corresponding eigenvalue. It is interesting to note that the eigenvalues resulting from a perturbation of $\varepsilon$ are contained within the contour of the resolvent given by $\left\|(i \omega \mathrm{l}-\mathrm{L})^{-1}\right\| \geq \varepsilon^{-1}$, see [8].

Exercise: Derive a link between a bound on the maximum excursion of a perturbed eigenvalue from its unperturbed location for a perturbation of norm $\varepsilon$ and the resolvent norm contour of $\varepsilon^{-1}$. Verify your results numerically.

\subsection{Adjoint modes}

In the previous section we have seen how the eigenvector $\mathbf{p}$ of $\mathrm{L}^{H}$, the matrix adjoint to $\mathrm{L}$, provides information 
(a)

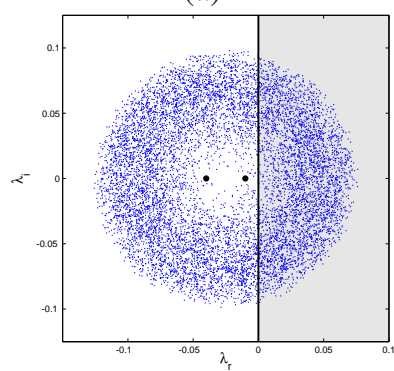

(b)

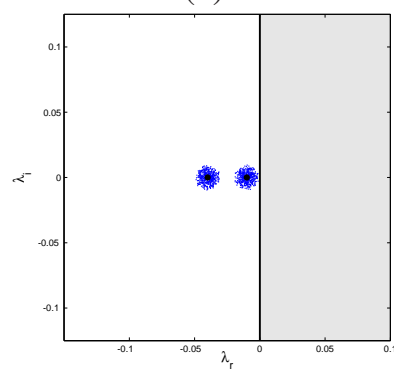

Fig. 15. Sensitivity of eigenvalues, illustrated on the $2 \times 2$-model problem. (left) Superposition of 100 spectra of the perturbed nonnormal system matrix with $\mu=1$; (right) same for the normal system matrix with $\mu=0$. In both cases, the norm of the perturbation matrix is $\|\Delta L\|=10^{-2}$.

about the system's sensitivity. We next show how the adjoint eigenvalue problem and, more generally, solutions of the adjoint system can be used to study the sensitivity of the underlying flow to external and internal perturbations.

An important property of the adjoint modes has been treated in [1]. Given two vectors we define an inner product as $\langle\mathbf{p}, \mathbf{q}\rangle=\mathbf{p}^{H} \mathbf{q}$, from which we derive that the transpose complex-conjugate matrix $\mathrm{L}^{H}$ satisfies $\langle\mathbf{p}, \mathbf{L} \mathbf{q}\rangle=\mathbf{p}^{H} \mathbf{L q}=$ $\left\langle\mathrm{L}^{H} \mathbf{p}, \mathbf{q}\right\rangle$ which also provides a definition of the adjoint matrix $\mathrm{L}^{H}$

Exercise: Compute the matrix adjoint to $\mathrm{L}$ associated with the weighted inner product $\langle\mathbf{p}, \mathbf{q}\rangle=\mathbf{p}^{H} \mathbf{Q q}$.

From the above definition, we obtain

$$
\langle\mathbf{p}, \mathbf{L q}\rangle=\mathbf{p}^{H} \mathrm{QLq}=\mathbf{p}^{H} \mathrm{QLQ}{ }^{-1} \mathrm{Q} \mathbf{q},
$$

where the adjoint matrix $\mathrm{L}^{+}$is given by $\mathrm{L}^{+}=\left(\mathrm{QLQ}^{-1}\right)^{H}=$ $\mathrm{Q}^{-1} \mathrm{~L}^{H} \mathrm{Q}$.

If we consider the eigenpairs $\left(\mathbf{q}_{i}, \lambda_{i}\right)$ and $\left(\mathbf{p}_{j}, \lambda_{j}^{H}\right)$ of the matrix $L$ and its adjoint, it is straighforward to show that the eigenvalues of $\mathrm{L}^{H}$ and $\mathrm{L}$ are complex conjugate to each other. Starting with the identity $\left\langle\left(\lambda_{j}^{H}-L^{H}\right) \mathbf{p}_{j}, \mathbf{q}_{i}\right\rangle=0$, we derive

$$
\left\langle\mathbf{p}_{j},\left(\lambda_{j}-\mathrm{L}\right) \mathbf{q}_{i}\right\rangle=\left\langle\mathbf{p}_{j},\left(\lambda_{j}-\mathrm{L}-\lambda_{i}+\mathrm{L}\right) \mathbf{q}_{i}\right\rangle=0,
$$

where we applied the definition of the adjoint and added $0=$ $\left(\lambda_{i}-\mathrm{L}\right) \mathbf{q}_{i}$. After a few manipulations, we finally arrive at

$$
\left(\lambda_{j}-\lambda_{i}\right)\left\langle\mathbf{p}_{j}, \mathbf{q}_{i}\right\rangle=0 \quad \Longrightarrow \quad\left\langle\mathbf{p}_{j}, \mathbf{q}_{i}\right\rangle=\delta_{i j}
$$

The last expression, with $\delta_{i j}$ as the Kroenecker delta, establishes the so-called bi-orthogonality condition: the eigenmodes of the direct and adjoint matrix are orthogonal to each other, if they are not associated with the same eigenvalue. This condition can be exploited to project any initial condition or external forcing onto the basis formed by the system's eigenvectors.
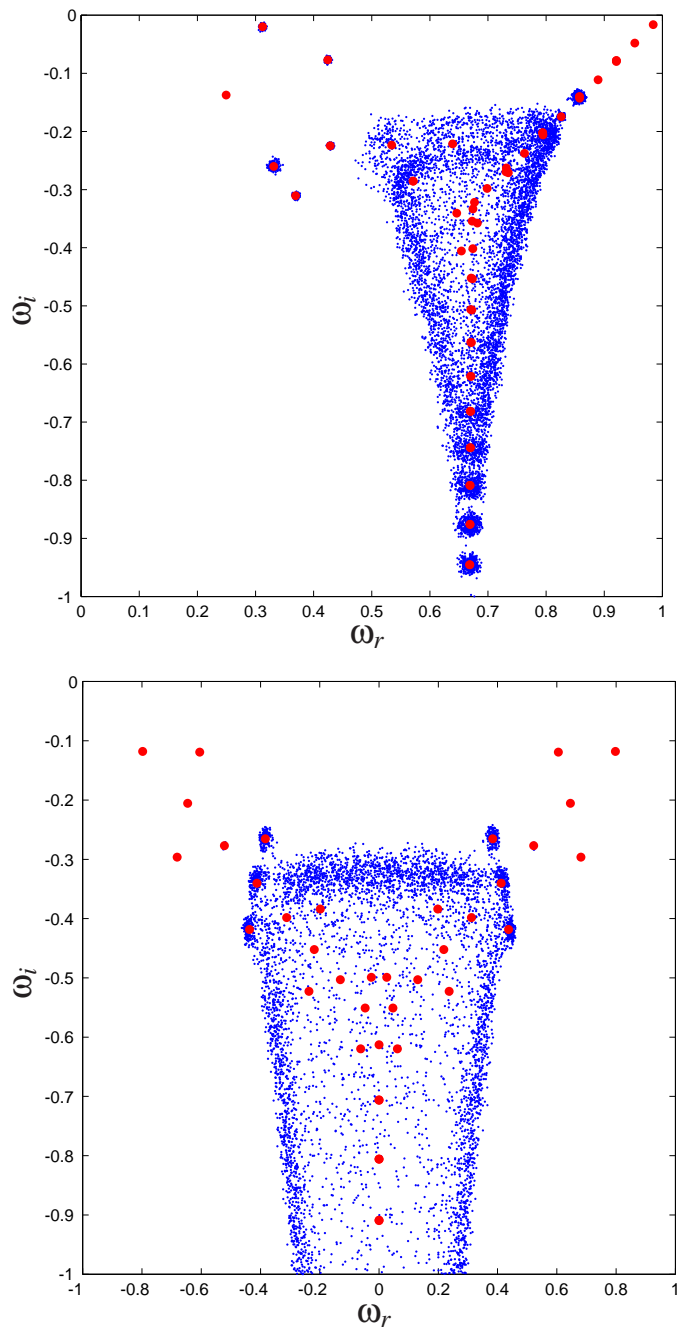

Fig. 16. Sensitivity of eigenvalues, for plane Poiseuille (left) and plane Couette (right) flow. The unperturbed spectrum is illustrated by red symbols. A superposition of 200 spectra (in blue) is shown for $\alpha=1, \beta=0$. The Poiseuille spectrum (for $R e=2000$ ) is perturbed by random matrices of norm $\varepsilon=5 \cdot 10^{-3}$. The Couette spectrum (for $\operatorname{Re}=1000$ ) is perturbed by random matrices of norm $\varepsilon=10^{-3}$. The resolvent norm can be displayed in the complex plane using the routine Resolvent.m.

\subsubsection{Sensitivity to initial conditions and forcing}

In many situations we are particularly interested in the sensitivity of eigenvalues to initial conditions or external forcing. Again, the adjoint solution is playing an important role. Let us consider the asymptotic behavior of the driven linear system

$$
\frac{d}{d t} \mathbf{q}=\mathrm{L} \mathbf{q}+\mathbf{f}
$$

with initial conditions $\mathbf{q}(0)=\mathbf{q}_{0}$ and external forcing $\mathbf{f}$. Applying the Laplace transform to (37) we obtain

$$
[\mathrm{L}+s \mathrm{l}] \cdot \hat{\mathbf{q}}=\hat{\mathbf{f}}(s)+\mathbf{q}_{0}
$$




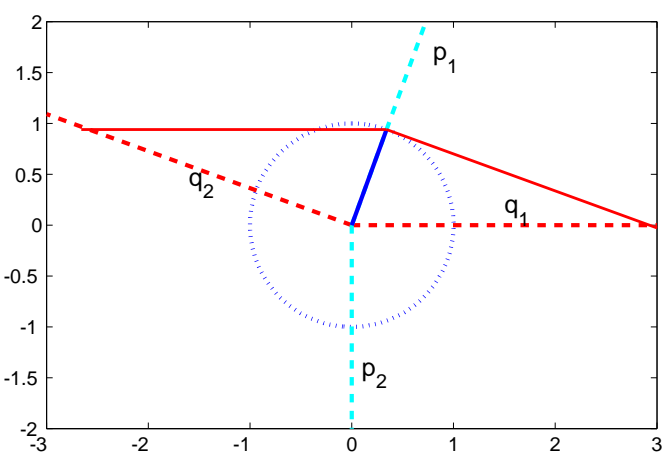

Fig. 17. Sketch of two non-orthogoonal eigendirections, $\mathbf{q}_{1}$ and $\mathbf{q}_{2}$, and the corresponding bi-orthogonal adjoint modes, $\mathbf{p}_{\mathbf{1}}$ and $\mathbf{p}_{\mathbf{2}}$. The figure displays how the adjoint mode provides the largest projection in the direction of the corresponding direct eigenvector.

with $s$ as the Laplace variable. The solution of (37) can then be formally written in terms of the inverse Laplace transform as

$$
\mathbf{q}(t)=\frac{1}{2 \pi \mathrm{i}} \int_{\gamma-\mathrm{i} \infty}^{\gamma+\mathrm{i} \infty}[\mathrm{L}+s \mathrm{l}]^{-1} \cdot\left(\hat{\mathbf{f}}(s)+\mathbf{q}_{0}\right) \exp (s t) \mathrm{d} s .
$$

We can further simplify this expression by rewriting the operator $[\mathrm{L}+s \mathrm{l}]^{-1}$ using its dyadic representation

$$
[\mathrm{L}+s \mid]^{-1}=\sum_{k} \frac{1}{\left(s-\lambda_{k}\right)} \frac{\mathbf{q}_{k} \mathbf{p}_{k}^{H}}{\mathbf{p}_{k}^{H} \mathbf{q}_{k}}
$$

where $\mathbf{p}_{k}$ and $\mathbf{q}_{k}$ are, respectively, the left and right eigenvectors of $L$ (corresponding to the eigenvalue $\lambda_{k}$ ) satisfying the equations

$$
\left[\mathrm{L}+\lambda_{k} \mathrm{I}\right] \mathbf{q}_{k}=0 \quad \mathbf{p}_{k}^{H}\left[\mathrm{~L}+\lambda_{k} \mathrm{l} \mathrm{I}\right]=0 .
$$

Using the residue theorem we obtain

$$
\begin{aligned}
\mathbf{q}(t)= & \frac{1}{2 \pi \mathrm{i}} \int_{\gamma-\mathrm{i} \infty}^{\gamma+\mathrm{i} \infty} \sum_{k} \frac{1}{\left(s-\lambda_{k}\right)} \frac{\mathbf{q}_{k} \mathbf{p}_{k}^{H}}{\mathbf{p}_{k}^{H} \mathbf{q}_{k}}, \\
& \left(\hat{\mathbf{f}}(s)+\mathbf{q}_{0}\right) \exp (s t) \mathrm{d} s, \\
= & \sum_{k} \frac{\mathbf{q}_{k} \mathbf{p}_{k}^{H}}{\mathbf{p}_{k}^{H} \mathbf{q}_{k}}\left(\hat{\mathbf{f}}\left(\lambda_{k}\right)+\mathbf{q}_{0}\right) \exp \left(\lambda_{k} t\right), \\
= & \sum_{k} A_{k} \mathbf{q}_{k} \exp \left(\lambda_{k} t\right),
\end{aligned}
$$

where the coefficients $A_{k}$, representing the amplitude of the modal expansion, are given by

$$
A_{k}=\frac{\mathbf{p}_{k}^{H}\left[\hat{\mathbf{f}}\left(\lambda_{k}\right)+\mathbf{q}_{0}\right]}{\mathbf{p}_{k}^{H} \mathbf{q}_{k}}=\frac{\mathbf{p}_{k}^{H} \hat{\mathbf{f}}\left(\lambda_{k}\right)+\mathbf{p}_{k}^{H} \mathbf{q}_{0}}{\mathbf{p}_{k}^{H} \mathbf{q}_{k}}
$$

This expression indicates how a specific mode is initialized by the initial condition $\mathbf{q}_{0}$ or by an external (Laplacetransformed) forcing $\hat{\mathbf{f}}\left(\lambda_{k}\right)$. The adjoint vector $\mathbf{p}_{k}$ is a determining factor in this expression; it can also be thought of as the variable that quantifies the influence of initial condition or external forcing on the temporal behavior of the solution (expressed in terms of an eigenvector expansion).

This result shows that the optimal way to introduce an unstable mode is not by initializing it at $t=0$ but, rather, to start with the adjoint mode. In the case of normal systems, these two modes coincide, while for non-normal systems they can differ substantially. In the latter case, using the adjoint mode as an initial condition maximizes the magnitude of the unstable mode while maintaining a specified initial energy, as shown in the sketch in figure 17. As an exercise the reader can verify that for an unstable system and for long optimization times (after all transients have died out) the optimal initial condition is indeed the adjoint of the unstable mode.

Exercise: Compute the adjoint of the least stable mode, e.g. for Poiseuille flow with $R e=10000, \alpha=1$ and $\beta=0$. Compute the optimal initial condition for the same configuration and large final time, $t_{f}=1000$. Compare the two results and explain what you observe.

\subsubsection{Sensitivity analysis using adjoint variables}

Next, a more general derivation is presented that provides more detail on the specific terms of the Navier-Stokes equations and their adjoint analogue. In particular, the role of adjoints in the description of sensitivity measures will be stressed. The following derivation is general and conceptually extends to more complex equations in a straightforward manner, although with a sometimes substantial increase in algebraic manipulations. In contrast to the previous section, we will abandon the modal expansion and examine general disturbances. To this end, we consider the continuous, linearized, incompressible Navier-Stokes equations in primitive variables according to

$$
\begin{aligned}
\frac{\partial \mathbf{u}}{\partial t}+L(U, R e) \mathbf{u}+\nabla p & =0 \\
\nabla \cdot \mathbf{u} & =0
\end{aligned}
$$

where the linear operator $L$ contains the base flow advection and diffusive terms and is given by $L(U, R e) \mathbf{u}=U \cdot \nabla \mathbf{u}+$ $\mathbf{u} \cdot \nabla U-\frac{1}{R e} \nabla^{2} \mathbf{u}$. Multiplying, respectively, by the differentiable vector and scalar fields $\mathbf{u}^{+}$and $p^{+}$and adding the two resulting expressions, we obtain

$$
\left(\frac{\partial \mathbf{u}}{\partial t}+L(U, R e) \mathbf{u}+\nabla p\right) \cdot \mathbf{u}^{+}+(\nabla \cdot \mathbf{u}) p^{+}=0
$$

Upon integration by parts over time and space, using a spatio-temporal inner product covering the spatial domain $\mathcal{D}$ 
and the time-interval $\left[\begin{array}{ll}0 & t\end{array}\right]$, we can write

$$
\begin{array}{r}
\int_{0}^{t} \int_{\mathcal{D}}\left[\left(\frac{\partial \mathbf{u}}{\partial t}+L(U, R e) \mathbf{u}+\nabla p\right) \cdot \mathbf{u}^{+}+(\nabla \cdot \mathbf{u}) p^{+}\right]= \\
-\int_{0}^{t} \int_{\mathcal{D}}\left[\mathbf{u} \cdot\left(\frac{\partial \mathbf{u}^{+}}{\partial t}+L^{+}(U, R e) \mathbf{u}^{+}+\nabla p^{+}\right)+p\left(\nabla \cdot \mathbf{u}^{+}\right)\right] \\
\int_{0}^{t} \frac{\partial \mathbf{u} \cdot \mathbf{u}^{+}}{\partial t}+\int_{\mathcal{D}} \nabla \cdot J=0,
\end{array}
$$

where the adjoint operator $L^{+}$is given by $L^{+}(U, R e) \mathbf{u}^{+}=$ $U \cdot \nabla \mathbf{u}^{+}-\nabla U \cdot \mathbf{u}^{+}+\frac{1}{R e} \nabla^{2} \mathbf{u}^{+}$and the so-called bilinear concomitant $J$

$$
J=U\left(\mathbf{u} \cdot \mathbf{u}^{+}\right)+\frac{1}{\operatorname{Re}}\left(\nabla \mathbf{u}^{+} \cdot \mathbf{u}-\nabla \mathbf{u} \cdot \mathbf{u}^{+}\right)+p^{+} \mathbf{u}+p \mathbf{u}^{+},
$$

arises during integration by parts from the exact differentials evaluated at the boundaries. Use of the divergence theorem in (46) gives the generalized Green's theorem for the linearized Navier-Stokes equations. We now assume that both $\mathbf{u}^{+}$and $p^{+}$satisfy the equations defined by the double integral above; boundary conditions for these variables can be chosen to simplify some of the boundary terms appearing in $J$. The adjoint equations thus read

$$
\begin{aligned}
\frac{\partial \mathbf{u}^{+}}{\partial t}+L^{+}(U, R e) \mathbf{u}^{+}+\nabla p^{+} & =0, \\
\nabla \cdot \mathbf{u}^{+} & =0 .
\end{aligned}
$$

The sign change in the diffusive term of $L^{+}$indicates that the above adjoint equations have to be integrated backwards in time to be well-defined.

Following the procedure of the previous section, we consider the same linearized Navier-Stokes equations defined by (44) with an external volume forcing $\mathbf{f}$ and a mass source $S$. Substituting into the expression above and integrating in time, we arrive at

$\mathbf{u}(t) \cdot \mathbf{u}^{+}(t)=\mathbf{u}(0) \cdot \mathbf{u}^{+}(0)+\int_{0}^{t} \int_{\mathcal{D}}\left(\mathbf{f} \cdot \mathbf{u}^{+}+S p^{+}\right)+\int_{\Gamma_{\mathcal{D}}} J \cdot n$.

If one then chooses the initial condition for the adjoint problem at time $t$ to be $\mathbf{u}^{+}(t)=\mathbf{u}(t)$, eq. (49) shows that the adjoint fields represent the effect of variations of the initial condition, external forcing and mass source on the energy of the perturbation at time $t$. In particular we see that the adjoint field $\mathbf{u}^{+}$acts as a filter for the initial condition $\mathbf{u}(0)$ and external forcing $\mathbf{f}$ whereas the so-called adjoint pressure $p^{+}$multiplies the mass source term. This implies that forcing and initial excitations are most efficient in those regions where the adjoint fields are largest, in agreement with the observation that the best way to trigger a specific mode is by using the velocity profile defined by its adjoint.
In the absence of external forcing $\mathbf{f}=0$ and mass sources/sinks $S=0$, we have $\mathbf{u}(t) \cdot \mathbf{u}^{+}(t)=\mathbf{u}(t) \cdot \mathbf{u}(t)=\mathbf{u}(0)$. $\mathbf{u}^{+}(0)$ and the largest variation of the disturbance at a prescribed final time $t$ is obtained when $\mathbf{u}(0)$ is parallel to $\mathbf{u}^{+}(0)$. In a more formal way, the gradient of the disturbance energy at some final time with respect to the initial condition is the terminal solution of the adjoint equation $\nabla_{\mathbf{u}(0)} \mathbf{u}^{2}(t)=\mathbf{u}^{+}(0)$. In the same way, the variations of the final energy with respect to any external forcing are given by the adjoint solution $\nabla_{\mathbf{f}} \mathbf{u}^{2}(t)=\mathbf{u}^{+}$and those with respect to mass sources by the adjoint pressure, $\nabla_{S} \mathbf{u}^{2}(t)=p^{+}$. The case of sensitivity to boundary conditions is slightly more complicated: it can be shown that in the case of non-homogeneous boundary conditions at a wall (e.g. wall-normal blowing and suction), we have

$$
\nabla_{\mathbf{u}_{\text {wall }}} \mathbf{u}^{2}(t)=\frac{1}{\operatorname{Re}} \nabla \mathbf{u}^{+}+p^{+} \cdot n
$$

with $n$ as the unit vector normal to the boundary. Equation (49) is general and valid for generic disturbances, not only for modal waves: the sensitivity map is obtained by a single calculation of the linearized Navier-Stokes equations and their adjoint. For practical purposes, one can iteratively determine the most dangerous initial condition and forcing by repeated time integration of (44) and (48), as shown, among others, in [9-11]. For a review of the use of adjoint equations for stability analysis the reader is also referred to the forthcoming review [12]. The approach introduced above can also be used to determine the effect of an external actuation on an instability.

Example: Determine the wall distribution of blowing and suction designed to reduce the growth of unstable TollmienSchlichting waves over a flat plate.

Assuming we have a two-dimensional solver for the direct and adjoint Navier-Stokes equations linearized about a spatially evolving boundary-layer flow, let us first integrate forward in time, with the unstable wave at the inflow, until the disturbance has reached the outflow boundary of the computational domain. We then choose the final field as the initial condition for the backward-in-time integration of the adjoint Navier-Stokes equations. The wall stress associated with the adjoint field, (50), then indicates where, when and how to actuate at the wall to decrease/increase the disturbance energy at the final time. This procedure provides information about the location and timing that yields the largest effect on the disturbance evolution. See [11].

\subsection{Parametric sensitivity}

It should be apparent to the reader that the above framework is far more flexible than demonstrated so far. Changes in initial conditions, external forces and wallblowing/suction strategies are not the only variations of interest. In particular, we often are interested in internal perturbations, i.e., perturbation of the operator, rather than external perturbations, i.e., perturbations in anything other than the governing equations. Examples of the former kind would be responses to changes in a wavenumber, the Reynolds number (or any other non-dimensional parameter) or even the base 
flow. For these types of changes, the response in, e.g., the eigenvalue is given by a weighted inner product of the direct and adjoint mode; the weight is given by a scalar or an operator. As an example, the response in an eigenvalue $\lambda_{j}$ due to a change in the Reynolds number is given by (33) as

$$
\Delta \lambda_{j}=\frac{\mathbf{p}_{j}^{H} \Delta \mathrm{L} \mathbf{q}_{j}}{\mathbf{p}_{j}^{H} \mathbf{q}_{j}}=\frac{\mathbf{p}_{j}^{H}\left(k^{2}-\mathrm{D}^{2}\right) \mathbf{q}_{j}}{\mathbf{p}_{j}^{H} \mathbf{q}_{j}} \Delta R e .
$$

In a similar fashion, the change in eigenvalue with respect to a change in wavenumber can be determined, i.e., $\Delta \lambda / \Delta \alpha$, which is related to the group velocity of a perturbation and forms the foundation of an analysis of absolute or convective instabilities in shear flows. The formulation involving the adjoint can be used to circumvent the more cumbersome deformation of Fourier-integration paths and the location of pinch-points in the complex plane.

\subsection{Structural sensitivity}

It is often instructive to determine the location in the computational domain that can be thought of as the origin of an instability. This location is characterized by both a high sensitivity of and a strong response in the most unstable mode. It thus should not come as a surprise that both the modal shape of the direct mode (measuring response) and the shape of the associated adjoint mode (measuring sensitivity) enter into the analysis of the origin of an instability.

\subsubsection{The origin of an instability}

In an attempt to gain physical understanding of an instability, the concept of a wavemaker, which locates the origin of an instability, can be introduced. This concept identifies the spatial location where a structured modification in the governing equations produces the largest drift of an eigenvalue. In our case, this coincides with determining the region where feedback from the velocity field to the external force is most effective. The derivation underlying this analysis is briefly outlined here for the discrete case. Further details and the corresponding derivation for continuous operators can be found in [13]. We start by introducing a feedback forcing in our linear system

$$
\frac{\partial \mathbf{q}}{\partial t}=L \mathbf{q}+\mathbf{f}=L \mathbf{q}+M \mathbf{q}
$$

by taking the external forcing $\mathbf{f}$ proportional to the state vector $\mathbf{q}$ via a matrix M. Recalling expression (33) we can state

$$
\Delta \lambda=\frac{\mathbf{p}^{H} \Delta \mathrm{L} \mathbf{q}}{\mathbf{p}^{H} \mathbf{q}}=\frac{\Delta \mathrm{L}: \mathbf{p} \mathbf{q}^{H}}{\mathbf{p}^{H} \mathbf{q}}
$$

and, identifying $\Delta \mathrm{L}=\mathrm{M}$, we define the structural sensitivity $\mathrm{S}$ as

$$
\mathrm{S}=\frac{\Delta \lambda}{\Delta \mathrm{L}}=\frac{\mathbf{p q}^{H}}{\mathbf{p}^{H} \mathbf{q}}
$$

which represents a matrix. If we consider a structural perturbation, spatially localized at $\mathbf{x}_{n}$ with a force proportional to the local velocity, $\mathrm{M}=\delta\left(\mathbf{x}-\mathbf{x}_{n}\right) \mathbf{q}$, we deduce that $S$ represents the sensitivity to a feedback forcing in a discrete point $\mathbf{x}_{n}$ of the vector $\mathbf{q}$; in our discretized system, $\mathbf{x}_{n}$ simplifies to a specific wall-normal location. Different norms of the matrix $\mathrm{S}$ can be used to build a spatial sensitivity map.

Exercise: Use the Matlab codes provided with this article and compute the structural sensitivity of the unstable mode for plane Poiseuille flow $(\operatorname{Re}=10000, \alpha=1)$. Do not forget to use the energy weight matrix when computing the adjoint matrix $\mathrm{L}^{+}=\mathrm{Q}^{-1} \mathrm{~L}^{H} \mathrm{Q}$ (see exercise above) and use equation (29) to extract the different velocity components. Compare the value of the base flow $U(y)$ at the location of maximum sensitivity with the phase speed $c=\omega / \alpha$ of the unstable mode.

\subsubsection{Sensitivity to base-flow modifications}

An even more challenging and interesting application of the adjoint-based sensitivity framework involves an assessment of the response of an eigenvalue with respect to changes in the base flow [14]. This problem is closely related to passive flow control, as it addresses the issue of a particular base-flow modification to avoid or delay the onset of modal perturbation growth on the modified base flow $[15,16]$. When studying the structural sensitivity to base-flow modifications we specify the type of variations of the matrix $L$ used in the derivation of (53). Within a linear context, a variation of the eigenvalue can be caused not only by a structural perturbation of the linearized equation but also via a variation of the base flow $U$.

In what follows, we outline a procedure to obtain an eigenvalue sensitivity when the base-flow modifications are brought about by a small-amplitude steady forcing of the Navier-Stokes equations; we do not assume any generic modification. We start by assuming

$$
\Delta \mathrm{L}=\frac{\delta \mathrm{L}}{\delta U} \Delta U
$$

to express (53) as

$$
\Delta \lambda=\frac{\mathbf{p}^{H} \frac{\delta \mathrm{L}}{\delta U} \Delta U \mathbf{q}}{\mathbf{p}^{H} \mathbf{q}} .
$$

To specify the type of base-flow variation, we assume that $U$ is a solution of the Navier-Stokes equations with a small, steady structural perturbation proportional to the local velocity $C U$. Mathematically, we have $\mathcal{N}(U)=C U$, with $\mathcal{N}$ as the Navier-Stokes operator. Taking the first variation of the 
expression above (in the limit of small perturbations), the following relation is obtained for the base-flow modification

$$
\frac{\delta \mathcal{N}}{\delta U} \Delta U \approx C U \quad \Longrightarrow \quad \Delta U=\left(\frac{\delta \mathcal{N}}{\delta U}\right)^{-1} \mathrm{C} U
$$

where the forcing $\mathrm{C} U$ is constant. The structural sensitivity to base-flow variations can then be recast by combining (55) and (56) to yield

$$
\Delta \lambda=\frac{\mathbf{p}^{H} \frac{\delta \mathrm{L}}{\delta U}\left(\frac{\delta \mathcal{N}}{\delta U}\right)^{-1} \mathrm{C} U \mathbf{q}}{\mathbf{p}^{H} \mathbf{q}} .
$$

Similarly to the case of the structural sensitivity to perturbations, when the feedback forcing is assumed to be localized in space, the sensitivity becomes a spatial map given by a dyadic product. The structural sensitivity presented in the previous section assumes a local force proportional to the local perturbation velocity, that is, a time-periodic forcing with the frequency of the instability mode. In contrast, the sensitivity considered here assumes a local force proportional to the local base-flow velocity, that is, a steady forcing inducing small base-flow deformations.

We now use the previous results to demonstrate and validate a passive control strategy for the flow around a cylinder. In principle, several approaches can be used to control the flow behind a bluff body. A very simple one was suggested by Strykowski \& Sreenivasan [17] who introduced a small control cylinder in the wake of the main cylinder. The effect of a small control cylinder on the flow field can be studied in terms of a structural sensitivity analysis as outlined above. The placement of a small cylinder of diameter $d^{*}$ in the near wake of a bluff body effectively results in a reaction force acting on the fluid which modifies the flow field and leads to a shift of the eigenvalue $\lambda$. Since the control cylinder is small, its presence can be thought of as a localized structural perturbation of the governing equations representing a localized feedback from velocity to force. In the limit of an infinitesimal small control cylinder, the local reacting force can be modeled by the first term of the Lamb-Oseen expansion for the drag of a cylinder in a creeping flow according to

$$
f=\delta \mathrm{O}=\frac{4 \pi}{\operatorname{Re} \ln \left(\frac{7.4}{R e_{c}}\right)}
$$

where $\delta O$ depends on the Reynolds number Re of the flow under investigation and on the Reynolds number of the small control cylinder $R e_{c}$ based on its diameter and the local velocity. The expression for $f$ represents pure resistance, i.e., a force whose direction is locally aligned with the local velocity vector. This means that the local feedback matrix is diagonal with elements of equal magnitude $\delta O$.

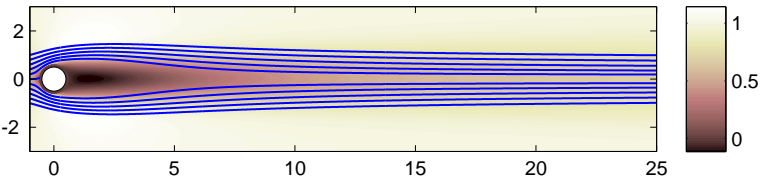

Fig. 18. Streamlines and velocity magnitude for the base flow past a circular cylinder at $R e=50$.

It is important to note that the structural perturbation acts both at the perturbation level by modifying in a direct way the structure of the eigenvalue problem and at the base-flow level by perturbing the steady base-flow which in turn affects the linearized Navier-Stokes operator. In order to build a spatial map which can be used as a guideline for a control strategy, both effects must be taken into consideration, i.e., the total eigenvalue drift is the sum of the two contributions: structural sensitivity to base-flow modifications and the structural sensitivity to perturbations (53). By inspecting the real and imaginary part of the sensitivity measure, it is possible to quantify the effect of the structural perturbation on the growth rate and the frequency of the unstable mode, respectively. A more detailed review of these concepts is provided in Sipp et al. [18].

\subsection{A complete analysis of a global instability}

Before discussing various extensions of the receptivity and sensitivity analysis introduced so far, we will work through the different steps of an inclusive receptivity/sensitivity analysis associated with a flow instability. To this end, we introduce the concept of global modes [19]. Many relevant flow configurations are characterized by multiple inhomogeneous coordinate directions; therefore, one cannot use the Fourier transform to reduce the governing equations to only one inhomogeneous direction, as is the case for plane channel flows introduced above. Rather, the eigenfunctions and the optimal initial conditions/forcing depend on more than one coordinate direction. In other words, while in parallel wall-bounded shear flows (such as channel flow) we seek solutions of the form $\tilde{\mathbf{u}}(x, y, z, t)=\mathbf{u}(y) \exp [\mathrm{i}(\alpha x+\beta z-\omega t)]$, in more general geometries we are interested in solutions expressed as $\tilde{\mathbf{u}}(x, y, z, t)=\mathbf{u}(\mathbf{x}) \exp (-\mathrm{i} \omega t)$.

For simplicity, we will consider as a test case the twodimensional flow past a circular cylinder: this flow is known to undergo a first bifurcation from a two-dimensional steady state $U(x, y)$ to a two-dimensional periodic limit cycle as the Reynolds number, based on the free-stream velocity and the cylinder diameter, increases above $R e_{c}=47$. The analysis is presented for a modal instability: a supercritical Hopf bifurcation. A similar approach can be followed for a globally stable system characterized by large transient growths (see [20] for an example of the latter).

Example: Analysis of the first bifurcation of incompressible flow past a circular cylinder. 

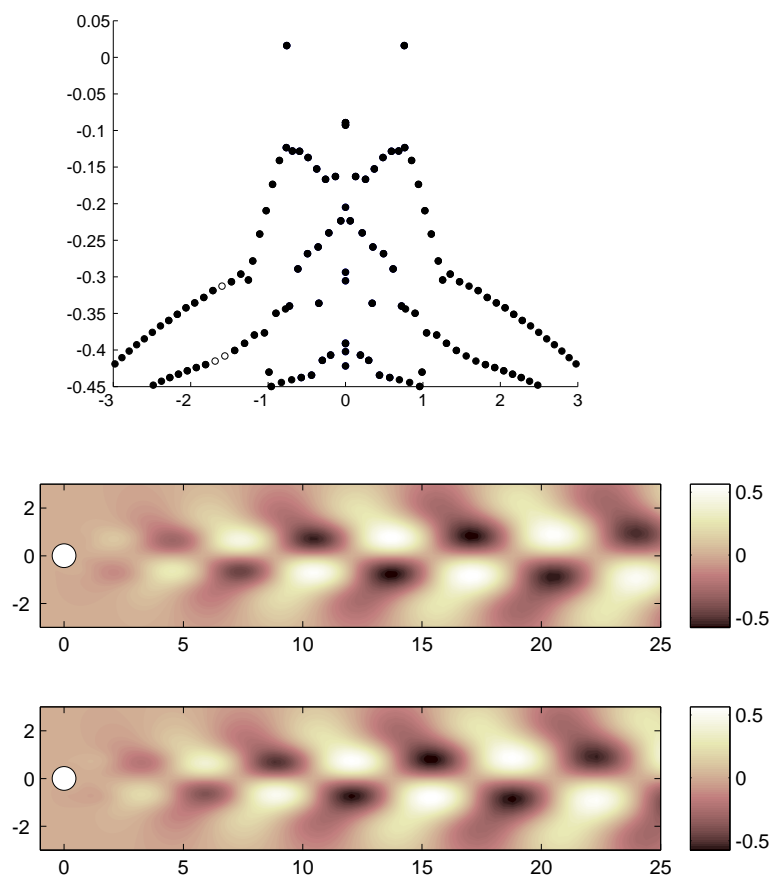

Fig. 19. (Top) Spectrum of eigenvalues and (bottom) unstable global mode, real and imaginary part, for the flow past a circular cylinder at $R e=50$.

\section{Base flow}

The first step consists of the computation of a base flow, see figure 18. The steady base flow cannot be computed by timemarching the Navier-Stokes equations due to a global instability for Reynolds numbers larger than $R e=47$. In such cases, one can resort to Newton iterations or, in some cases, exploit the symmetry of the instabilities. In our case, this would amount to considering only the upper half of the domain and impose symmetry conditions at the centerline: under this setup, asymmetric unstable modes are not feasible, and the flow will eventually converge to a steady state. More recently, a procedure denoted as selective frequency damping (SFD) has been proposed to eliminate instabilities by low-pass filtering the velocity field; this technique causes the filtered simulations to eventually converge to a steady state [21]. This approach allows us to use an existing Navier-Stokes solver and still adopt a time-marching procedure.

\section{Modal analysis}

The second step includes the computation of the eigenvalues (spectrum) and eigenmodes of the system. The spectrum for flow around a cylinder at $R e=50$ and the corresponding unstable global mode are illustrated in figure 19. Note the appearance of two unstable eigenvalues characterized by the same absolute value of the frequency owing to the time-invariance of the problem. The shape of the global mode displayed in the lower panel of the same figure shows the shedding of a traveling mode behind the cylinder. Only a part of the computational domain is shown, and care has to be exercised regarding its truncation to arrive at converged results.

\section{Adjoint modes and receptivity}

As discussed above, the adjoint global mode represents the initial
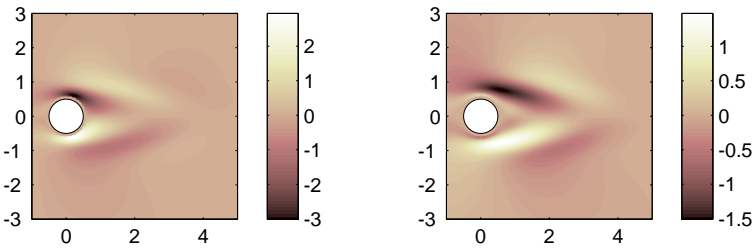

Fig. 20. Adjoint of the unstable global mode (real and imaginary part) for flow past a circular cylinder at $R e=50$.
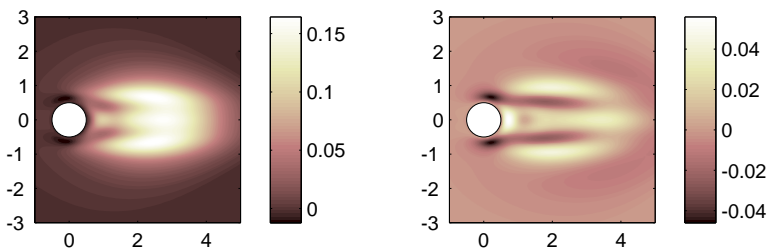

Fig. 21. Structural sensitivity (the wave maker) for flow past a circular cylinder at $R e=50$. Variations of the real and imaginary part of the eigenvalue are reported in the figure.

condition that projects maximally onto the associated global mode. In addition, its maximum reveals the location in the flow domain where an external forcing most efficiently triggers the corresponding global mode. The adjoint mode, associated with the unstable global mode, is shown in figure 20 for flow past a circular cylinder at $R e=50$. Interestingly, the regions most receptive to external forcing are located on the sides of the cylinder and inside the recirculation zone. Non-negligible receptivity to external forcing is also found upstream of the cylinder.

\section{Structural sensitivity: the wave maker}

The point-wise product of adjoint and direct mode provides information about the region in space where a feedback-forcing proportional to the local velocity has the largest impact on the eigenvalue drift. This region is referred to as the core (or origin) of the instability or the wave maker: since small structural perturbations of the system will have the largest impact on the instability, this is the region in space where the instability originates. Due to the fact that the system eigenvalues are complex numbers, the sensitivity can be divided into real and imaginary part, corresponding to variations of the frequency of the unstable mode and of its growth rate. The wave maker for the first bifurcation of flow past a circular cylinder is depicted in figure 21. It is located symmetrically near the re-circulation zones behind the cylinder.

\section{Sensitivity to base-flow modifications}

Finally, we display the sensitivity to base-flow modifications, figure 22. The top panel displays the shift of the real and imaginary part of the least stable eigenvalue in the presence of base-flow modifications induced by a purely resistive force, proportional to the local base flow and directed against it. Comparing to the structural sensitivity to external perturbations (see figure 21), we observe that modifications of the base flow are most effective, if implemented near the boundaries of the re-circulation region, somewhat farther away from the symmetry axis. The bottom panels in the figure show 

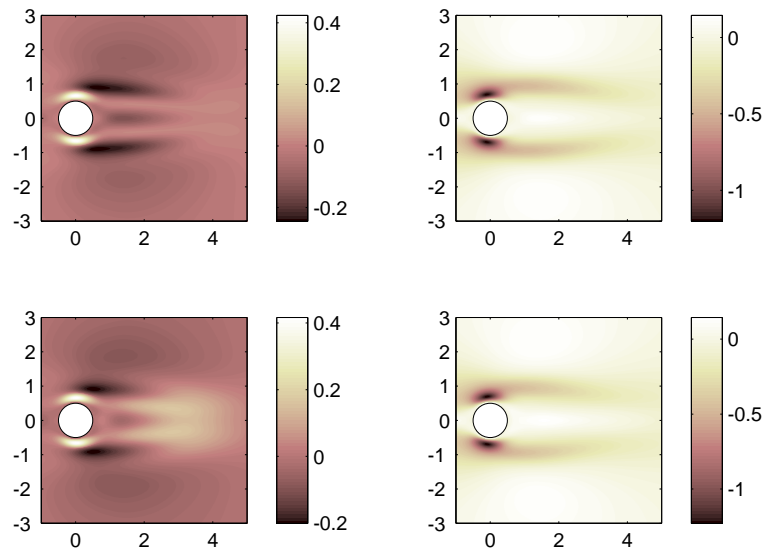

Fig. 22. Sensitivity to base-flow modifications and total sensitivity to a localized resistance force for flow past a circular cylinder at $R e=$ 50. Variations of the real and imaginary part of the eigenvalue are reported in the figure.

the total eigenvalue shift, as induced by base-flow modifications due to a feedback forcing proportional to the local mean velocity and by a structural feedback proportional to the local perturbation velocity. This type of analysis provides first suggestions on the passive control of an instability: by forcing the system with a smaller control cylinder, strategically placed in the wake of the principal one, we are able to optimally delay the onset of vortex shedding and thus stabilize the flow. It is important to keep in mind that the sensitivity analysis is targeting the least stable mode. For a complete analysis of the effect of passive control strategies, one also has to verify that formerly stable modes are not destabilized by the proposed actuation.

\section{Extensions}

The techniques, tools and methodology described in this article provides a more complete and encompassing treatment of linear fluid systems. Even though we have demonstrated these techniques on toy models and rather generic flow configurations (plane Couette flow, plane Poiseuille flow, flow around a cylinder), they apply — in principle to flows with more complexity, inhomogeneous dimensions and complicated flow behavior. While generalizing the techniques above to these situations exceeds the scope of this article, we shall briefly outline the extensions most commonly encountered in fluid systems.

\subsection{Computing global modes}

Most problems dealing with the stability of fluid flow have inhomogeneous directions and in general take place in a more complex geometry than those covered in this article. The theoretical framework introduced so far is unchanged in the latter case; however, difficulties arise due to the substantial computational costs associated with significantly larger system matrices.

From a physical point of view, it is interesting to note that when dealing with open, spatially evolving flows, e.g.,
Table 2. Computational storage cost for the state vector $\mathbf{q}$ and the system matrix $\mathrm{L}$ and operation count for stability problems with one and two inhomogeneous directions. $N$ : number of grid points in one coordinate direction.

\begin{tabular}{lclc}
\hline case & $\mathbf{q}$ & $\mathrm{L}$ & Operation count \\
\hline $\mathbf{q}(x)$ & $O(N)$ & $O\left(N^{2}\right)$ & $O\left(N^{3}\right)$ \\
$\mathbf{q}(x, y)$ & $O\left(N^{2}\right)$ & $O\left(N^{4}\right)$ & $O\left(N^{6}\right)$ \\
\hline
\end{tabular}

in the presence of an inhomogeneous streamwise direction, a new source of non-normality appears: the so-called streamwise non-normality [22], in contrast to the case of channel flows where the non-normality is defined as componentwise. In non-normal systems, the direct and adjoint eigenvectors are not parallel and their inner product tends to become very small. In parallel shear flows, direct and adjoint modes are varied in different velocity components, as reflected by the fact that the optimal initial condition and response consist of different velocity components: dominantly wall-normal velocity for the initial condition and dominantly wall-normal vorticity, parallel to the streamwise velocity, for the flow response. In open flows, on the other hand, direct and adjoint global modes have different spatial support (typically, upstream for the adjoint mode and downstream for the direct mode) so that their overlap is limited and their inner product small. This feature reflects the spatial amplification of unstable waves, which eventually decay or leave the computational domain.

In table 2 we list the increase of storage and computational time associated with a stability problem where two coordinate directions are inhomogeneous. In the case of multiple inhomogeneous directions, direct eigenvalue algorithms quickly become prohibitively expensive, and one has to resort to iterative algorithms such as the Arnoldi technique [23].

The idea underlying the Arnoldi procedure is to reduce the system by approximating the action of the linear operator in an orthonormal basis $\mathrm{V}$, see figure 23. By repeated application of the system matrix $L$ to a starting vector, a Krylov subspace is formed, which, after orthonormalization, is used to reduced the stability matrix $L$ to a low-dimensional representation, the upper Hessenberg matrix $\mathrm{H}$. Some of the eigenvalues of the original matrix are approximated by those of the reduced-order matrix, i.e., $\operatorname{eig}(H) \subset \operatorname{eig}(L)$. The Arnoldi technique can be applied to any matrix whose action of a given vector can be computed in a convenient way, e.g., the exponential matrix $\exp (t \mathrm{~L})$ for modal stability problems, or the symmetric matrix $\exp (t \mathrm{~L}) \exp \left(t \mathrm{~L}^{H}\right)$, whose eigenvalues are the singular values of the exponential matrix, for transient growth analyses.

For completeness, the algorithm below lists the code segment necessary to build the $k$-th column $v_{k}$ in $\mathrm{V}$ and the entries in the matrix $\mathrm{H}$, given the previous set of columns $\left\{v_{1}, \ldots, v_{k-1}\right\}$. 

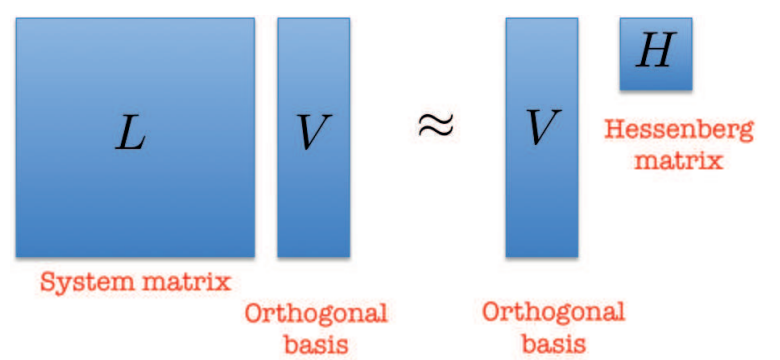

Orthogonal basis

Fig. 23. Sketch of the numerical procedure used to approximate a matrix $L$ by the Hessenberg matrix $H$ using the orthonormal basis $V$.

$$
\begin{aligned}
& v_{k}=\mathrm{L} v_{k-1} \\
& \text { for } j=1: k-1 \\
& \qquad \mathrm{H}_{j, k-1}=\left\langle v_{j}, v_{k}\right\rangle \\
& \quad v_{k}=v_{k}-\mathrm{H}_{j, k-1} v_{j} \\
& \text { end } \\
& \mathrm{H}_{k, k-1}=\left\|v_{k}\right\| \\
& v_{k}=v_{k} / \mathrm{H}_{k, k-1}
\end{aligned}
$$

The Arnoldi algorithm above has been used to compute the global modes for the flow past a circular cylinder used in the example of the previous section. For other examples of global modes describing features of flows in complex geometries, the reader is referred to [19,24-26], among others.

\subsection{Generalization to time-dependent base flows}

The base flow about which we have linearized our governing equations has been assumed to be time-independent, yielding a linear time-invariant (LTI) system. In many applications, however, the base flow is time-dependent. Startup or inlet flows or flows produced by a periodic or non-periodic pressure gradient (such as in pumps or turbomachinery) are examples where the time-invariance assumption does not hold for the base flow and $U=U(\mathbf{x}, t)$. Linearization about a time-varying base flow yields a time-varying system matrix $\mathrm{L}(t)$. In this case, we cannot express the solution of the initial-value problem in the form of a matrix exponential. Nonetheless, we can introduce the concept of a propagator $\mathrm{P}(t)$, a linear operator that maps the initial condition $\mathbf{q}_{0}$ to the perturbation $\mathbf{q}$ at a later time $t$ according to

$$
\mathbf{q}(t)=\mathrm{P}(t) \mathbf{q}_{0} .
$$

As before, we seek the largest possible energy amplification, optimized over all admissible initial disturbances, using the energy-based inner product $\langle\cdot, \cdot\rangle_{E}$, and arrive at

$$
\begin{aligned}
& G(t)=\max _{\mathbf{q}_{0}} \frac{\langle\mathbf{q}, \mathbf{q}\rangle_{E}}{\left\langle\mathbf{q}_{0}, \mathbf{q}_{0}\right\rangle_{E}}=\max _{\mathbf{q}_{0}} \frac{\left\langle\mathrm{P}(t) \mathbf{q}_{0}, \mathrm{P}(t) \mathbf{q}_{0}\right\rangle_{E}}{\left\langle\mathbf{q}_{0}, \mathbf{q}_{0}\right\rangle_{E}}= \\
& \max _{\mathbf{q}_{0}} \frac{\left\langle\mathrm{P}^{H}(t) \mathrm{P}(t) \mathbf{q}_{0}, \mathbf{q}_{0}\right\rangle_{E}}{\left\langle\mathbf{q}_{0}, \mathbf{q}_{0}\right\rangle_{E}} .
\end{aligned}
$$

$$
\mathbf{q}_{0}^{(n+1)}=\rho^{(n)} \mathbf{P}^{H} \mathrm{Pq}_{\mathbf{o}^{(n)}}
$$

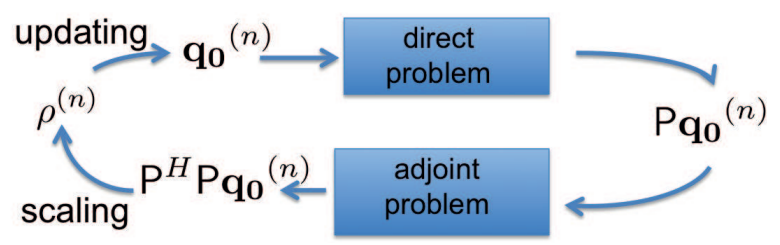

Fig. 24. Sketch of adjoint looping procedure. Power iterations are used to determine the principal eigenvector and eigenvalue corresponding to the optimal initial condition and growth for any timedependent flow. The propagator $P$ evolves the initial condition from $t=0$ to some final time $t$, while $\mathrm{P}^{H}$ backpropagates a terminal vector from $t$ to $t=0$ using the adjoint equation.

From this last expression we see that the optimal amplification is given by the largest eigenvalue of the symmetric (normal) matrix $\mathrm{P}^{H}(t) \mathrm{P}(t)$. The principal eigenvector and eigenvalue can be found simply by power iteration, see figure 24 , but the Arnoldi procedure is also a viable alternative, if more than one mode is sought.

The largest eigenvalue is obtained by iteratively applying the composite mapping $\mathrm{P}^{H} \mathrm{P}$ according to

$$
\mathbf{q}_{0}^{(n+1)}=\rho^{(n)} \mathrm{P}^{H} \mathrm{P} \mathbf{q}_{0}^{(n)} .
$$

We note that in general the matrices $\mathrm{P}(t)$ and $\mathrm{P}^{H}(t)$ are not explicitly available for time-dependent flows; however, their action on a vector $\mathbf{q}_{0}$ can be computed straightforwardly. To this end, we proceed in two steps

$$
\mathbf{w}=\mathrm{P} \mathbf{q}_{0}^{(n)}, \quad \mathbf{q}_{0}^{(n+1)}=\rho^{(n)} \mathrm{P}^{H} \mathbf{w} .
$$

This two-step process amounts to propagating an initial condition forward in time over a given interval $[0, t]$ after which the adjoint initial condition, $\mathbf{w}$, is evolved backward in time. At the end of each iteration, the initial condition is scaled to unit energy by the factor $\rho^{(n)}$. Convergence of the adjoint looping process can be assessed by monitoring the relative variations of $\rho^{(n)}$ or of $\mathbf{q}_{0}^{(n)}$. It is apparent that this iterative technique (adjoint looping) can be applied to any general time-dependent fluid system and thus allows the treatment of a far larger class of stability problems.

\subsection{Link to constrained optimization}

We have seen that the optimal growth problem can be reformulated in variational form as a constrained optimization problem. This fact offers the possibility to enforce a great variety of constraints and governing equations, even nonlinear equations as will be discussed briefly below. The general idea of constrained optimization is shortly introduced here; more details can be found in the respective tutorial of this issue and in [27]. 
To demonstrate the potential of this approach we consider a slightly modified problem and take the time integral of the disturbance energy from $t=0$ to some time $t_{f}$ as our cost objective. We introduce a functional $J=\{\langle\mathbf{q}, \mathbf{q}\rangle\}$ where $\{\cdot\}$ indicates integration in time over the interval $\left[0, t_{f}\right]$. Our constraint requires that $\mathbf{q}$ is a solution of the linearized Navier-Stokes equations, written here for simplicity as $d \mathbf{q} / d t-\mathbf{L} \mathbf{q}=0$, including conservation of mass. Instead of direct substitution of this constraint into the cost functional (using the matrix exponential for LTI systems), we enforce the governing equation via a Lagrange multiplier $\mathbf{q}^{+}$. This has the advantage that the solution of the governing equations does not need to be known explicitly. The second condition we need to impose is the initial perturbation energy, which we normalize to one for the linear case considered here. We thus have

$$
\mathcal{L}=J-\left\langle\mathbf{q}^{+}, \frac{d}{d t} \mathbf{q}-\mathbf{L} \mathbf{q}\right\rangle-\sigma\left(\left\langle\mathbf{q}_{0}, \mathbf{q}_{0}\right\rangle-1\right) .
$$

The functional $\mathcal{L}$ reads $\mathcal{L}\left(\mathbf{q}, \mathbf{q}^{+}, \mathbf{q}_{0}, \sigma\right)$, and its first variations must be zero at optimality. The first two variations simply restate the imposed constraints

$$
\begin{aligned}
\frac{\delta \mathcal{L}}{\delta \mathbf{q}^{+}}=0 & =\frac{d}{d t} \mathbf{q}-\mathrm{L} \mathbf{q}, \\
\frac{\delta \mathcal{L}}{\delta \sigma}=0 & =\left\langle\mathbf{q}_{0}, \mathbf{q}_{0}\right\rangle-1 .
\end{aligned}
$$

The variations with respect to the disturbance field $\mathbf{q}$ and the initial condition $\mathbf{q}_{0}$ can be obtained using integration by parts

$$
\begin{aligned}
\mathcal{L}= & J-\left\langle\mathbf{q}^{+}\left(t_{f}\right), \mathbf{q}\left(t_{f}\right)\right\rangle+\left\langle\mathbf{q}_{0}^{+}, \mathbf{q}\right\rangle+ \\
& \left\langle-\frac{d}{d t} \mathbf{q}^{+}-\mathrm{L}^{H} \mathbf{q}, \mathbf{q}\right\rangle-\sigma\left(\left\langle\mathbf{q}_{0}, \mathbf{q}_{0}\right\rangle-1\right),
\end{aligned}
$$

where we assume a zero initial condition $\mathbf{q}^{+}\left(t_{f}\right)$ for the adjoint problem. The remaining optimality conditions follows as

$$
\frac{\delta \mathcal{L}}{\delta \mathbf{q}}=-\frac{d}{d t} \mathbf{q}^{+}-\mathrm{L}^{H} \mathbf{q}^{+}+2 \mathbf{q}, \quad \frac{\delta \mathcal{L}}{\delta \mathbf{q}_{0}}=\mathbf{q}_{0}^{+}-2 \sigma \mathbf{q}_{0}
$$

The first expression above defines the adjoint problem pertaining to our objective functional (the time-integral of the perturbation energy). We particularly stress the fact that the adjoint system is forced by the solution of the direct problem q. From a practical point of view, this implies that the solution must be stored and used when solving the adjoint problem. The second relation in (66) dictates that the initial condition of the direct problem should fall parallel to the adjoint solution at final time $t=0$. The optimal initial condition, satisfying (64) and (66), can be obtained iteratively as described below.

(i) Given an initial guess $\mathbf{q}_{0}^{(n)}$, we march the governing equations forward in time and retain the solution $\mathbf{q}^{(n)}(t)$. (ii) We solve the adjoint problem defined in (64) with homogenous initial conditions and forcings $\mathbf{q}^{(n)}(t)$.

(iii) We rescale the final solution of the adjoint problem to obtain a next (and improved) guess $\mathbf{q}_{0}^{(n+1)}=$ $\mathbf{q}_{0}^{+(n)} /\left\langle\mathbf{q}_{0}^{+(n)}, \mathbf{q}_{0}^{+(n)}\right\rangle$.

(iv) If $\left|\mathbf{q}_{0}^{(n+1)}-\mathbf{q}_{0}^{(n)}\right|>\varepsilon$, we proceed to step (i); $\varepsilon$ as a given, user-specified tolerance.

Exercise: Show that the variational form of the optimization problem defined in section 3, maximum possible disturbance energy at final time over initial disturbance energy, results in the conditions obtained for general time-dependent system and that these are solved by (61) as in figure 24 .

\subsection{Nonlinear analysis}

We would like to conclude this review with a brief reference to a full nonlinear analysis. A variational formulation is most appropriate in this case as the governing equations to be added as a constraint are not limited to linear equations but can also consist of the nonlinear Navier-Stokes equations. Nonlinear optimals in canonical wall-bounded shear flows have recently been the object of several research efforts [28-30]. Adding the nonlinear Navier-Stokes equations to the augmented cost functional, the resulting adjoint system is still linear, since the adjoint variable enters linearly into the augmented cost functional. However, regardless of the objective functional considered, the solution of the forward problem appears in the adjoint system in the form of variable coefficients. This can be most easily seen by considering the first variation of the nonlinear convective term of the incompressible Navier-Stokes equations. We have

$$
\left\langle\mathbf{u}^{+}, \mathbf{u} \nabla \mathbf{u}\right\rangle \quad \Longrightarrow \quad\left\langle-\mathbf{u} \nabla \mathbf{u}^{+}, \delta \mathbf{u}\right\rangle
$$

Therefore, the flow fields from the forward sweep have to be stored and inserted during the backward sweep. For large systems and long optimization horizons, these storage requirements may become restrictive. In this case, a checkpointing procedure can be used which stores direct solutions at only a few, strategically placed, instants (checkpoints) in time; during the backward sweep, these checkpoint solutions will be used as initial conditions to recover the direct solutions between two consecutive checkpoints which then will be inserted in the backward sweep. The extra effort in additional forward simulations translates into a benefit of reduced storage requirements. Recent studies have concentrated on an optimal placement of the checkpoints which exploits the available memory resources in the best possible manner during the optimization procedure. Once checkpointing has been taken into account, the adjoint looping procedure discussed in the previous two sections is still applicable in the nonlinear regime.

As an example of a nonlinear optimization, we summarize the formulation adopted in [30]. In this work the objective functional is the time-averaged dissipation

$$
J=\frac{1}{T} \int_{0}^{T} \frac{1}{R e} \nabla \mathbf{u}: \nabla \mathbf{u} d t
$$


and the augmented Lagrange functional reads

$$
\begin{gathered}
\mathcal{L}=J-\int_{0}^{T}\left\langle\mathbf{u}^{+}, N S(\mathbf{u})\right\rangle+\left\langle p^{+}, \nabla \cdot \mathbf{u}\right\rangle d t- \\
\sigma\left[\left\langle\left(\mathbf{u}_{0}-U\right),\left(\mathbf{u}_{0}-U\right)\right\rangle-\varepsilon_{0}\right]
\end{gathered}
$$

where $\mathbf{u}^{+}, p^{+}, \sigma$ are the Lagrange multipliers, i.e., the adjoint variables. The nonlinear Navier-Stokes equations have been abbreviated as $N S$, and $\varepsilon_{0}$ stands for the initial kinetic energy of the perturbation. As before, the velocity vector and the base flow are denoted by $\mathbf{u}$ and $U$, respectively.

Exercise: Given the variational form defined by (69) and (68), show that the linear system for the adjoint variable $\mathbf{u}^{+}$ is forced by the term

$$
-\frac{1}{T \operatorname{Re}} \nabla^{2} \mathbf{u}
$$

\section{Summary and conclusions}

We have presented a suite of tools and techniques for the analysis of fluid systems as to their stability, receptivity and sensitivity. An argument for the replacement of eigenvaluebased, modal analysis in favor of an optimization-based, nonmodal approach has been made, and methods have been introduced to quantify the response to initial conditions, to external harmonic forcing, to a time-dependent base flow, and to localized base-flow modifications. A variational framework has proven flexible and efficient in addressing relevant questions related to the linear (and nonlinear) behavior of fluid systems. In particular, adjoint variables - carrying sensitivity and gradient information — play an important role in the analysis. It is hoped that this more complete description of stability, receptivity and sensitivity characteristics of fluid flow will become more commonplace in the fluid dynamics community; this article is intended to support this effort.

The reader is encouraged to work through the exercises and examples. Matlab-codes for most of the figures in this article are supplied at the NORDITA-website and are intended to provide an easy and accessible introduction to the concepts of this tutorial article. They should give researchers and graduate students interested in these techniques a natural starting point for their own studies of the stability, receptivity and sensitivity of fluid systems.

\section{Acknowledgements}

For discussions on structural sensitivity, we wish to thank Flavio Giannetti, Jan Pralits and Paolo Luchini. The help of Iman Lashgari for the figures in section 5 is gratefully acknowledged.

\section{References}

[1] Schmid, P. J., and Henningson, D. S., 2001. Stability and Transition in Shear Flows. Springer, New York.

[2] Schmid, P. J., 2007. "Nonmodal stability theory". Ann. Rev. Fluid Mech., 39, pp. 129-162.
[3] Horn, R. A., and Johnson, C. R., 1994. Topics in Matrix Analysis. Cambridge University Press, Cambridge.

[4] Trefethen, L. N., Trefethen, A. N., Reddy, S. C., and Driscoll, T. A., 1993. "Hydrodynamic stability without eigenvalues”. Science, 261, pp. 578-584.

[5] Jovanovic, M. R., and Bamieh, B., 2005. "Componentwise energy amplification in channel flows". J. Fluid Mech., 534, pp. 145-184.

[6] Klinkenberg, J., deLange, H. C., and Brandt, L., 2011. "Modal and nonmodal stability of particle-laden channel flow”. Phys. Fluids, 23(064110).

[7] Saffman, P. G., 1962. "On the stability of laminar flow of a dusty gas". J. Fluid Mech., 13, pp. 120-128.

[8] Trefethen, L. N., and Embree, M., 2005. Spectra and Pseudospectra. Princeton University Press, Princeton.

[9] Andersson, P., Berggren, M., and Henningson, D. S., 1999. "Optimal disturbances and bypass transition in boundary layers". Phys. Fluids, 11, pp. 134-150.

[10] Luchini, P., 2000. "Reynolds-number independent instability of the boundary layer over a flat surface. Part 2: Optimal perturbations". J. Fluid Mech., 404, pp. 289309.

[11] Pralits, J. O., Airiau, C., Hanifi, A., and Henningson, D. S., 2000. "Sensitivity analysis using adjoint parabolized stability equations for compressible flows". Flow Turbulence and Combustion, 65, pp. 321-346.

[12] Luchini, P., and Bottaro, A., 2014. "Adjoint equations in stability analysis". Annu. Rev. Fluid Mech., 46.

[13] Giannetti, F., and Luchini, P., 2007. "Structtural sensitivity of the first instability of the cylinder wake". $J$. Fluid Mech., 581, pp. 167-197.

[14] Bottaro, A., Corbett, P., and Luchini, P., 2003. "The effect of base flow variationon flow stability". J. Fluid Mech., 476, pp. 293-302.

[15] Marquet, O., Sipp, D., and Jacquin, L., 2008. "Sensitivity analysis and passive control of cylinder flow". $J$. Fluid Mech., 615, pp. 221-252.

[16] Pralits, J. O., Brandt, L., and Giannetti, F., 2010. "Instability and sensitivity of the flow around a rotating circular cylinder". J. Fluid Mech., 650, pp. 513-536.

[17] Strykowski, P. J., and Sreenivasan, K. R., 1990. "On the formation and suppression of vortex 'shedding' at low Reynolds number”. J. Fluid Mech., 218, pp. 71-107.

[18] Sipp, D., Marquet, O., Meliga, P., and Barbagallo, A., 2010. "Dynamics and control of global instabilities in open flows: a linearized approach". Appl. Mech. Reviews, 63, p. 030801.

[19] Theofilis, V., 2011. "Global linear instability". Annu. Rev. Fluid Mech., 43, pp. 319-352.

[20] Brandt, L., Sipp, D., Pralits, J. O., and Marquet, O., 2011. "Effect of base-flow variation in noise amplifiers: the flat-plate boundary layer". J. Fluid Mech., 687, pp. 503-528.

[21] Åkervik, E., Brandt, L., Henningson, D. S., Hœpffner, J., Marxen, O., and Schlatter, P., 2006. "Steady solutions of the Navier-Stokes equations by selective frequency damping". Phys. Fluids, 18, p. 068102.

[22] Chomaz, J. M., 2005. "Global instabilities in spatially 
developing flows: non-normality and nonlinearity". Annu. Rev. Fluid Mech., 37, pp. 357-392.

[23] Trefethen, L. N., and Bau, D., 1997. Numerical Linear Algebra. SIAM, Philadelphia.

[24] Mack, C. J., Schmid, P. J., and Sesterhenn, J. L., 2008. "Global stability of swept flow around a parabolic body: connecting attachment-line and crossflow modes". J. Fluid Mech., 611, pp. 205-214.

[25] Mack, C. J., and Schmid, P. J., 2011. "Global stability of swept flow around a parabolic body: features of the global spectrum". J. Fluid Mech., 669, pp. 375-396.

[26] Bagheri, S., Schlatter, P., Schmid, P. J., and Henningson, D. S., 2009. "Global stability of a jet in crossflow". J. Fluid Mech., 624, pp. 33-44.

[27] Gunzburger, M. D., 2003. Perspectives in Flow Control and Optimization. SIAM.

[28] Pringle, C. C. T., and Kerswell, R. R., 2010. "Using nonlinear transient growth to construct the minimal seed for shear flow turbulence". Phys. Rev. Lett., 105(154502).

[29] Cherubini, S., De Palma, P., Robinet, J.-C., and Bottaro, A., 2010. "Rapid path to transition via nonlinear localized optimal perturbations in a boundary-layer flow". Phys. Rev. E, 82(066302).

[30] Monokrousos, A., Bottaro, A., Brandt, L., Vita, A. D., and Henningson, D. S., 2011. "Non-equilibrium thermodynamics and the optimal path to turbulence in shear flows". Phys. Rev. Lett., 106(134502). 